\title{
Properties and the origin of Almost Monoenergetic Ion (AMI) beams observed near the Earth's bow shock
}

\author{
V. N. Lutsenko and E. A. Gavrilova \\ Space Research Institute RAS, Profsoyuznaya 84/32, Moscow, Russia
}

Received: 26 November 2010 - Revised: 15 July 2011 - Accepted: 1 August 2011 - Published: 29 August 2011

\begin{abstract}
Beams of Almost Monoenergetic Ions (AMI) in the energy range from 20 to $800 \mathrm{keV}$ were discovered in the DOK-2 experiment (Interball project) in the magnetosheath and upstream of the Earth's bow shock. This work summarizes the analysis results of $\sim 730$ AMI events registered in 1995-2000. Statistics of AMI properties, their nature and origin are considered. The analysis of a large array of new data confirmed our earlier suggested ideas on the AMI nature, origin, and their acceleration model. These ideas were further developed and refined. According to this model, AMI are a result of solar wind ions acceleration in small regions with a potential electric field arising due to disruptions of the bow shock current sheet filaments. It has been found that the reason of the current filaments disruptions in most cases was the Hot Flow Anomaly phenomenon (HFA) caused by an interaction of a tangential discontinuity in the solar wind with the Earth's bow shock. It is shown that the study of AMI can provide new information on large-scale properties and dynamics of the bow shock current sheet.
\end{abstract}

Keywords. Magnetospheric physics (Current systems; Solar wind-magnetosphere interactions) - Space plasma physics (Charged particle motion and acceleration)

\section{Introduction}

Ions and electrons accelerated in the near-Earth plasma in the energy range of $20 \mathrm{keV}$ to $1000 \mathrm{keV}$ usually have smooth spectra with a negative slope. Particle acceleration is commonly obliged to inductive electric fields emerging as a consequence of magnetic fields variations in the magnetosphere and on its borders. Broad humps can arise in spectra when

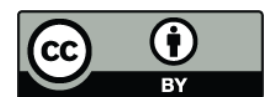

Correspondence to: V. N. Lutsenko (vlutsenk@iki.rssi.ru) magnetospheric energetic particles gain additional acceleration near the quasi-perpendicular bow shock (Anderson, 1981). Rather narrow peaks can be observed by particles propagation due to time-of-flight or gradient-drift dispersions. Energies of such peaks which were cut out from the original smooth spectrum vary with time and an observation site (Lutsenko et al., 2005, 2008). Almost monoenergetic electrons with energies of 1 to $10 \mathrm{keV}$ were often observed in the auroral zone. They possibly have obtained their energy after passing electrostatic double layers (see e.g. Alfvén, 1977, and references therein). However, in the very sources of energetic ions and electrons in the energy range of 20 $1000 \mathrm{keV}$, we always had to deal with smooth spectra, often having power-law or exponential forms.

In 1995-2000, the DOK-2 experiment (Lutsenko et al., 1998) was carried out within the framework of the Interball project on both Tail (Interball-1) and Auroral (Interball-2) spacecrafts (S/C). Interball-1 was launched on 8 March 1995 and worked until 16 October 2000; its orbit had an apogee of $31.14 R_{\mathrm{E}}$, a perigee of $1.16 R_{\mathrm{E}}$, an orbital plane inclination to the equator of $62.8^{\circ}$, and a period of 4 days. The distinctive characteristics of the DOK-2 spectrometer were record high energy and time resolutions. The instrument used 56-channel logarithmic analyzers to measure ion spectra in the range of $20-800 \mathrm{keV}$ and electron spectra in the range of $25-400 \mathrm{keV}$. It allowed to discover in the Earth's magnetosphere and near its borders the beams of Almost Monoenergetic Ions (AMI) (Lutsenko and Kudela, 1999; Lutsenko, 2001), Fine Dispersion Structures (FDS) (Lutsenko et al., 2005, 2008), and a number of other phenomena (see e.g. Lutsenko et al., 2002), which could not be discovered earlier because of an insufficient energy resolution of the used instruments.

AMI were named so because their spectra contain 2 to 3 lines having the Gaussian shape, with relative values of full width at half maximum (FWHW) for the first line $d E 1 / E 1$ from 0.11 to 0.39 with a mean value of 0.25 . In the most general case we have observed events with 3 lines having

Published by Copernicus Publications on behalf of the European Geosciences Union. 
energy ratios of 1:2:(5-6). In events with lower intensities or with a higher background, only the first two lines with the energy ratio 1:2 were observed. Note that in energetic electron spectra measured with the same resolution narrow lines were observed only in the dispersion events (Lutsenko et al., 2005, 2008) associated with the acceleration processes in the plasma sheet of the magnetospheric tail. Except for two of our papers (Lutsenko and Kudela, 1999; Lutsenko, 2001) there have been no other publications on similar ion spectra until 2009. The first such publication appeared in 2009 (Klassen et al., 2009). The authors have used data from SEPT and SIT instruments (project STEREO). AMI were observed not only upstream and close to the Earth's bow shock, but also at distances up to $1900 R_{\mathrm{E}}$.

In our previous papers we have published results based on the analysis of the limited number of AMI events and suggested a hypothesis about their nature and origin. According to this hypothesis AMI are a result of solar wind ions acceleration by bursts of strong potential electric fields in small parts of current sheets in the magnetosphere and on its boundaries. These bursts arise due to disruptions of filaments forming these electric current sheets. After such disruption the electromotive force of the circuit (EMF) will be applied to a narrow disruption interval, creating a potential electric field with a magnitude up to $0.1 \mathrm{~V} \mathrm{~m}^{-1}$. For the neutral current sheet in the magnetotail, this process was observed directly at the acceleration place (Lutsenko et al., 2008). As to the magnetosheath and the region upstream of the bow shock where we had most of our AMI observations, a much greater number of events should be analyzed.

This article summarizes the results of study of the $\sim 730$ AMI events observed during the 5 years (1995-2000) in the solar wind (SW) and in the magnetosheath (MSH) near the Earth's bow shock (BS). Their properties, nature and origin are clarified. The earlier proposed model of AMI acceleration should be confirmed by the results of all the AMI events analysis, further developed and refined. In particular, the aims are:

1. To build the statistics of AMI line properties using the full set of our data, in particular for the ratio of numbers of accelerated protons and alpha-particles.

\section{To estimate angular distributions of AMI beams.}

3. To find a confirmation of our assumptions regarding nature of AMI lines in the results of other experiments.

4. To check our assumption on the mechanism of AMI acceleration by comparison of our observations with some consequences of the current layer filaments disruption model.

5. To make a calculation of ion trajectories in the acceleration region for estimation of its dimensions.
6. To find which current sheet is responsible for generation of AMI observed in the magnetosheath and upstream of the bow shock.

7. To find the reasons for current disruptions.

\section{Special features of DOK-2 that made possible the dis- covery and study of AMI. Confirmation of our hy- pothesis regarding nature of the lines in AMI spectra}

As it was noted in Sect. 1, the discovery and study of AMI became possible owing to the record high energy and time resolutions of the DOK-2 instrument (Lutsenko et al., 1998). The device was equipped with four telescopes for measuring parameters of energetic ions in the range of $20-800 \mathrm{keV}$ ( $1 \mathrm{p}$ and $2 \mathrm{p}$ ) and energetic electrons in the range of $25-400 \mathrm{keV}(1 \mathrm{e}$ and 2e). All telescopes used passively cooled Si-detectors with instrumental energy resolution $d E 1_{\text {ins }}=7-8 \mathrm{keV}$ and logarithmic pulse height analysers with 56 channels. The ion telescopes had aperture angles of $12.5^{\circ}$, the electron ones of $27^{\circ}$. Geometric factors of the telescopes were of $0.015 \mathrm{~cm}^{2} \mathrm{sr}$ for ion telescopes and of $0.066 \mathrm{~cm}^{2} \mathrm{sr}$ for electron ones. The axes of $1 \mathrm{p}$ and $1 \mathrm{e}$ telescopes were directed along the spin axis of the $\mathrm{S} / \mathrm{C}$ in the antisolar direction. The $2 \mathrm{p}$ and $2 \mathrm{e}$ telescopes were oriented in the solar hemisphere at an angle of $62^{\circ}$ to the S/C's spin axis directed toward the Sun. A spacecraft spin period was $\sim 2 \mathrm{~min}$.

The discovery and study of AMI was made possible not only because of the high energy resolution, but also through the use of a special adaptive measurement algorithm. The basis for the formation of the DOK-2 output data were Basic Measurements (BM) - complete 56-channel spectra measured every second. However, their transfer to ground receiving stations was possible only during the rare and short sessions of direct transmission since capacity of the onboard storage device was insufficient. To overcome this problem, two types of output information complementing each other were formed from BMs for each telescope:

1. Complete 56-channel spectra obtained by summing up individual BMs until any of the channels gets a certain number of particles $N_{\max }$ or accumulation time $d T$ reaches a certain value $T_{\max }$. These parameters could be changed by commands, but most often $T_{\max }=1280^{\circ} \mathrm{C}$ and $N_{\max }=10240$. As a result, at high particle fluxes the spectrum accumulation time was reduced to $2-5 \mathrm{~s}$, and at low fluxes it increased, but not more than Tmax.

2. Three parameters of temporal variations: TP1, TP2, and TP3 - each representing a sum of 4 consecutive spectrum channels. The accumulation time for TPs varied from 1 to $260 \mathrm{~s}$ and was determined by a special algorithm which ensured a reduction of the output information volume up to $10-100$ times, and at the same time retaining the ability to record all statistically significant variations in particle numbers in the TP's energy ranges. 

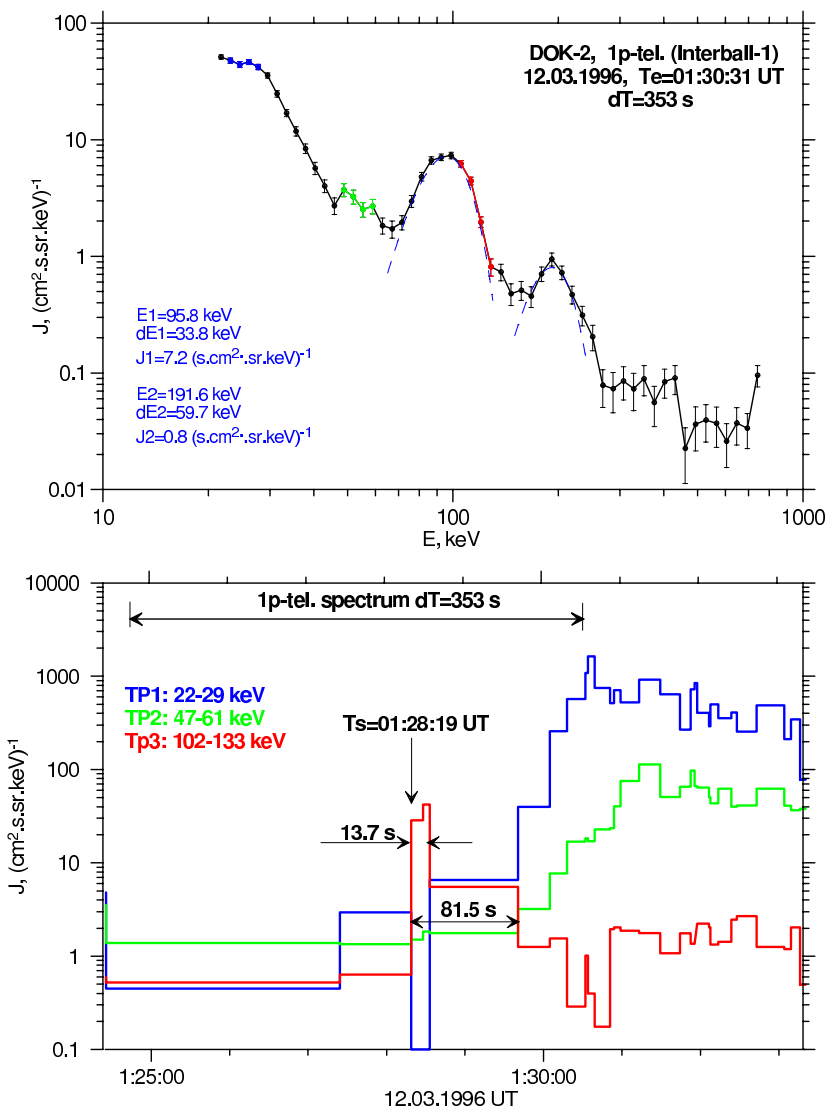

Fig. 1. An example of mutual complementarity of the full ion spectrum and the time profiles parameters TP. At the top - a full ion spectrum from 1p-telescope, measured in the solar wind and containing 2 AMI lines. Color markings show energy intervals of 3 TP-parameters. Here and below, $T_{\mathrm{e}}$ corresponds to the end of the spectrum accumulation interval, $d T$ to the accumulation duration. At the bottom - graphs of TP-parameters and the values of corresponding energies. For comparison, the spectrum accumulation time interval is also shown: $d T=353 \mathrm{~s}$.

Both of these types of output data are mutually complementary and proved to be very useful for searching and analyzing the data on AMI. Figure 1 gives an example of the AMI spectrum and corresponding TP-parameters measured in the solar wind on 12 March 1996 (1p-telescope). Three parts of the spectrum highlighted in blue, green, and red correspond to TP1, TP2, and TP3, respectively. The intensity of the AMI containing 2 lines was rather weak at a relatively high level of background ions with a usual smooth spectrum. Thus, the third line is almost invisible. As a result, the accumulation time for the spectrum turned out to be rather long and reached $353 \mathrm{~s}$. The energies $E$ and intensities $J$ at the maxima of the lines as well as their Full Width at Half Maximum (FWHM) $d E$ were determined by the Gaussian fitting (blue dashed lines and legends). The figure shows that the TP1 and TP2 intervals fall on the smooth background spectrum, while TP3 falls upon the first AMI line. Figure 1 (bot-

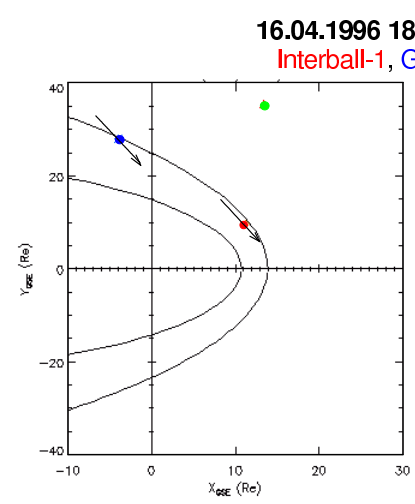

16.04.1996 18:30 - 19:00 UT

Interball-1, Geotail, Wind
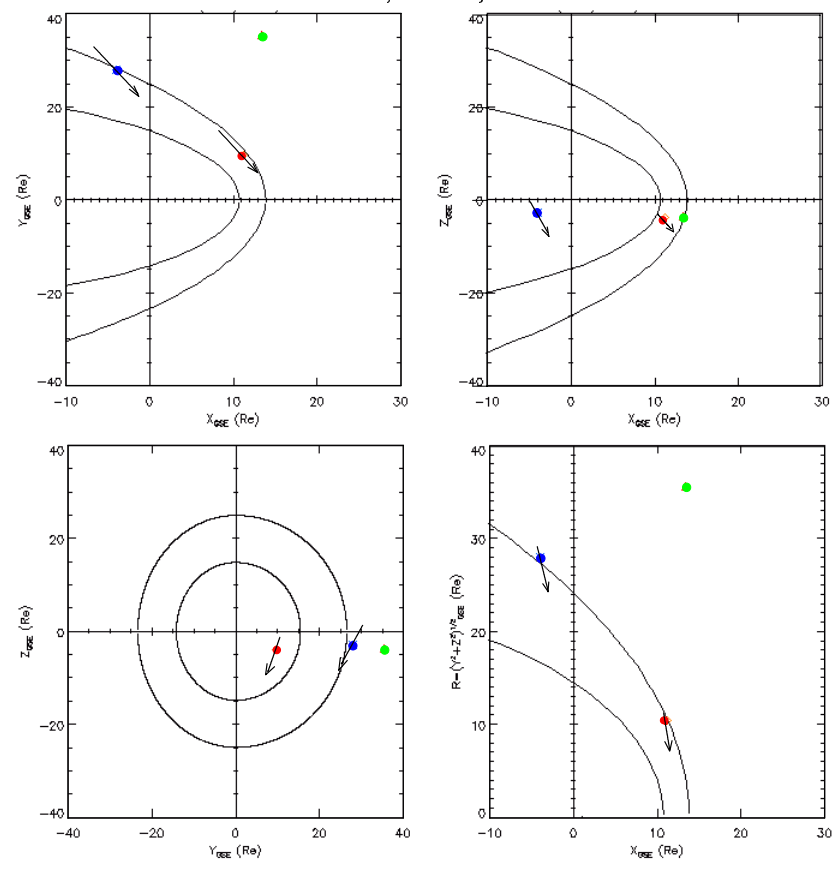

Fig. 2. Positions of Interball-1, Geotail and Wind S/C during the AMI event on 16 April 1996. Arrows show magnetic field directions on first two S/C.

tom graphs) shows time variations of TP1-TP3, the interval of the spectrum accumulation time and the energy values for TP1-TP3. Around 01:28:19 UT the TP3 intensity increases sharply and during the next $13.7 \mathrm{~s}$ exceeds the initial level by 46 times. Then, through $81.5 \mathrm{~s}$ after the increase it falls to approximately the initial level corresponding to the normal spectrum (TP3 is below TP2 and TP1). Thus, TPparameters allow to determine within $1 \mathrm{~s}$ accuracy the start time (01:28:19 UT) and duration (81.5 s) of the AMI event, which cannot be found only from the given spectrum in this case.

Although DOK-2 measured the energy spectra of all ions, doing so without charge and mass separation, the noted above relations of line energies allowed us to suppose that we deal with solar wind ions $\mathrm{H}^{+1}, \mathrm{He}^{+2}$, and the ion group $(\mathrm{C}, \mathrm{N}, \mathrm{O})^{+(5-6)}$ accelerated in some spatially confined potential electric field to energies proportional to their charges (Lutsenko and Kudela, 1999). We obtained a confirmation of this hypothesis in 2002, after a private communication of A. Lui and S. Nylund who sent us the detailed data from the EPIC instrument (Geotail) for several time intervals when Interball-1 and Geotail had been in the same physical region close enough to each other. The EPIC device (Williams et al., 1994) had $\sim 8$ times lower energy resolution but was able to measure separately the spectra of protons, alpha-particles, and ions of the (C, N, O) group. On 16 April 1996, two $\mathrm{S} / \mathrm{C}$ were in the MSH near the quasi-perpendicular BS (see 

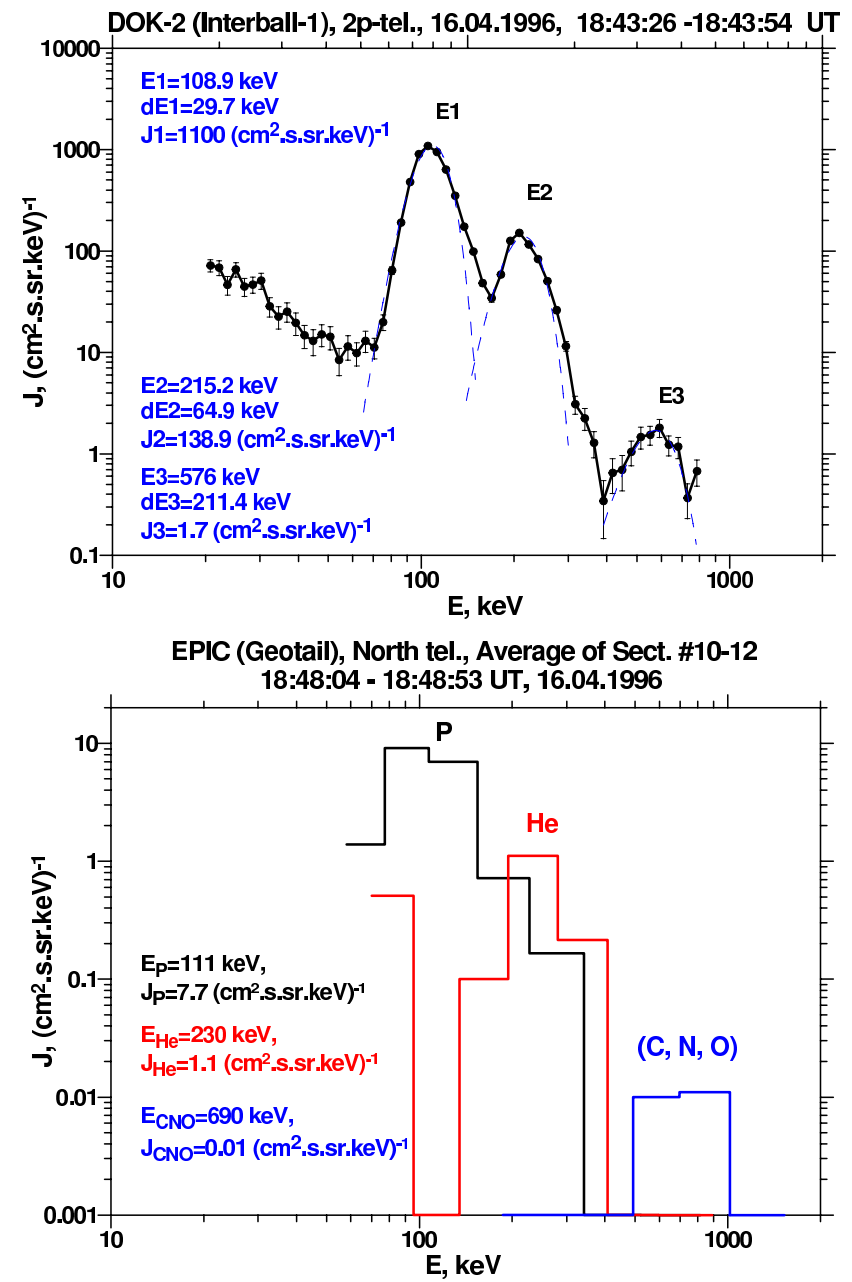

Fig. 3. Comparison of ion spectra measured on Interball-1 and Geotail in the MSH close to the BS during the AMI event on 16 April 1996. For DOK-2 (Interball-1) spectrum blue dashed lines and legends give results of Gaussian AMI lines fitting.

Fig. 2). The directions of the magnetic field on both satellites were approximately the same, so that both S/C were close to a magnetic connection.

Figure 3 compares the spectrum of all ions measured by DOK-2 (INTERBALL-1) with spectra of protons, alphaparticles, and the $(\mathrm{C}, \mathrm{N}, \mathrm{O})$ group measured by EPIC (Geotail). It is evident that 3 lines in the DOK-2 spectrum correspond to the peaks of protons, alpha-particles, and of the $(\mathrm{C}, \mathrm{N}, \mathrm{O})$ group in EPIC spectra. Ratios of the energies and intensities of 3 peaks in EPIC spectra are very close to the corresponding ratios in the DOK-2 spectrum.

After our first publications (Lutsenko and Kudela, 1999; Lutsenko, 2001), AMI were also found by Klassen et al. (2009) in the SEPT experiment (project STEREO) at distances up to $1100-1900 R_{\mathrm{E}}$ from the Earth. They present one more example of the identification of the first two AMI lines as ions of $\mathrm{H}^{+1}$ and $\mathrm{He}^{+2}$.
Table 1. Distribution of a total number of AMI events with 2-3 lines among DOK-2 telescopes and measurement regions.

\begin{tabular}{lccc}
\hline Telescope & SW & MSH & Total \\
\hline $1 \mathrm{p}$ & 314 & 38 & 352 \\
$2 \mathrm{p}$ & 187 & 183 & 370 \\
Total & 501 & 221 & 722 \\
\hline
\end{tabular}

\section{Where AMI events were observed}

AMI were observed by DOK-2 (Interball-1) mainly in the solar wind (SW) and magnetosheath (MSH) near the Earth's bowshock (BS), in all about 730 events. For our detailed analysis, the 722 most distinct AMI events with 2-3 lines in the spectrum were selected.

Table 1 shows how the number of observations is distributed between the 2 regions and 2 telescopes. Figure 4 shows the distribution of observation points in these regions. The extent of the observation region in the solar wind was limited by the $\mathrm{S} / \mathrm{C}$ apogee. Red dots correspond to the measurements, with the $1 \mathrm{p}$-telescope looking in the antisolar direction. Blue dots correspond to the measurements with the $2 p$-telescope directed toward the solar hemisphere at an angle of $62^{\circ}$ to the spin axis of the S/C. Distributions in Fig. 4 and Table 1 show that AMI propagating towards the Sun (1p) predominate in the SW, while in the MSH predominate AMI propagating from the BS (2p). It indicates that the AMI sources are located most probably on the BS, but not on the magnetopause, as it was supposed previously (Lutsenko, 2001).

Over the 5 years of Interball- 1 operation, the total DOK-2 observation time reached $18958 \mathrm{~h}$ in areas where these 722 AMI-events were observed. Thus, the average frequency of AMI-events observations in the SW and MSH was 0.038 per hour or 0.91 AMI-events per day. Since the observations at any moment were carried out only at one point, and AMI propagate as narrow beams, as we will shown further in Sect. 5, the real frequency of the AMI-events must be much higher.

AMI were also observed in the magnetotail plasma sheet where observations were complicated due to high-intensity background of energetic particles with the usual smooth spectra. These particles were generated together with AMI by the geotail current sheet filaments disruptions (Lutsenko et al., 2008). These AMI are not considered here.

\section{Statistics of AMI properties}

Figure 5 presents a histogram of AMI events durations. The exact determination of some specific event duration is not always possible, because sometimes two events overlap or follow directly one after another. When the data are obtained 

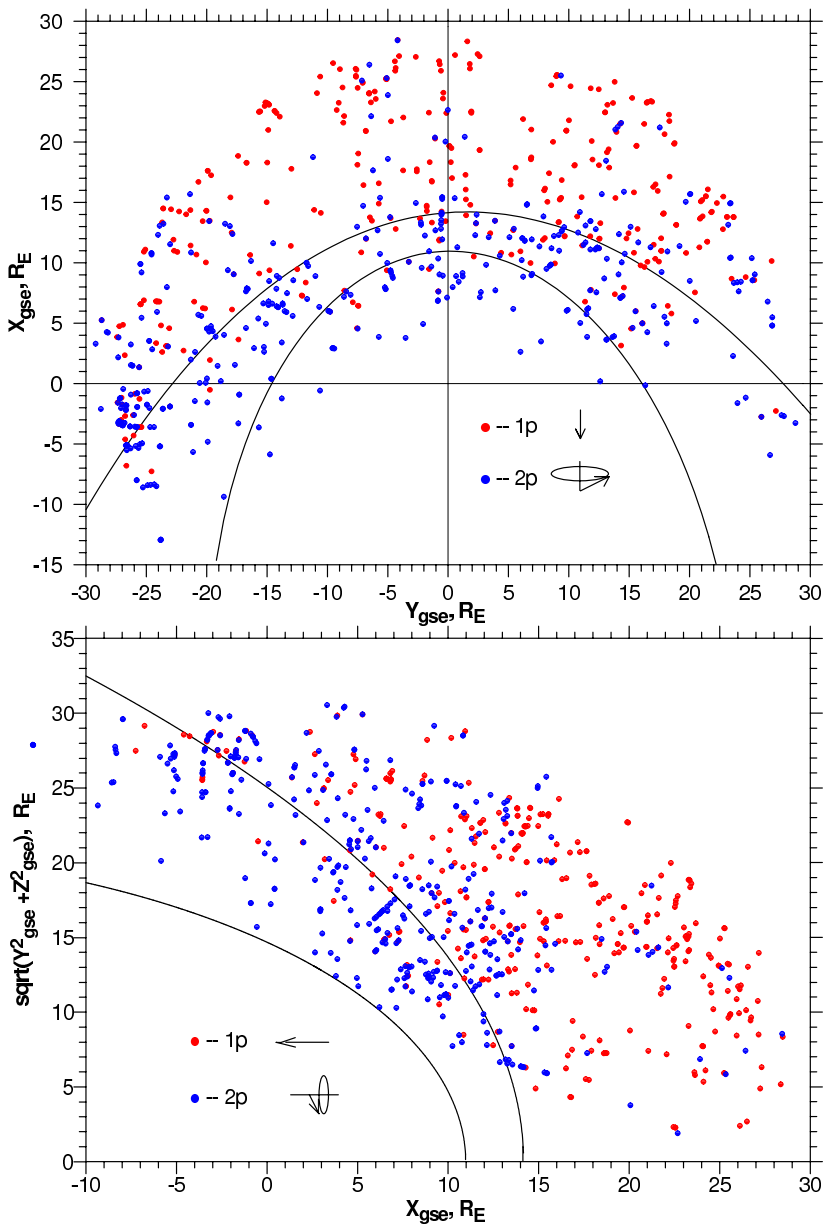

Fig. 4. Observation positions for 722 AMI events with 2-3 lines upstream of the BS and in the MSH (August 1995-August 2000).

Table 2. Statistical parameters of AMI beams (see Figs. 5-7).

\begin{tabular}{lccccc}
\hline $\begin{array}{l}\text { Parameter, } \\
\text { units }\end{array}$ & $\begin{array}{c}\text { Total numb. } \\
\text { of values }\end{array}$ & $\begin{array}{c}\text { Min. } \\
\text { value }\end{array}$ & $\begin{array}{c}\text { Max. } \\
\text { value }\end{array}$ & $\begin{array}{c}\text { Mean } \\
\text { value }\end{array}$ & $\begin{array}{c}\text { Std. } \\
\text { deviation }\end{array}$ \\
\hline Duration, s & 204 & 17 & 294 & 104.7 & 61.12 \\
$E 1, \mathrm{keV}$ & 722 & 25.8 & 409.9 & 94.6 & 59.56 \\
$E 2 / E 1$ & 722 & 1.52 & 2.91 & 2.034 & 0.158 \\
$E 3 / E 1$ & 114 & 3.68 & 7.13 & 5.25 & 0.698 \\
$d E 1_{\text {corr }} / E 1$ & 231 & 0.107 & 0.393 & 0.253 & 0.058 \\
$S_{\alpha} / S_{\mathrm{p}}$ & 154 & 0.34 & 66.77 & 12.35 & 13.24 \\
$N_{\alpha} / N_{\mathrm{p}}$ & 124 & 12.95 & 91.98 & 37.26 & 18.77 \\
\hline
\end{tabular}

from the 2p-telescope, the AMI beam often leaves the telescope field of view before the event ends because of the S/C rotation. Therefore, we used only the data from 1p-telescope and solely for those 204 AMI-events whose durations could be determined accurately enough. The statistical parameters of this and other distributions presented in this section are listed in Table 2.

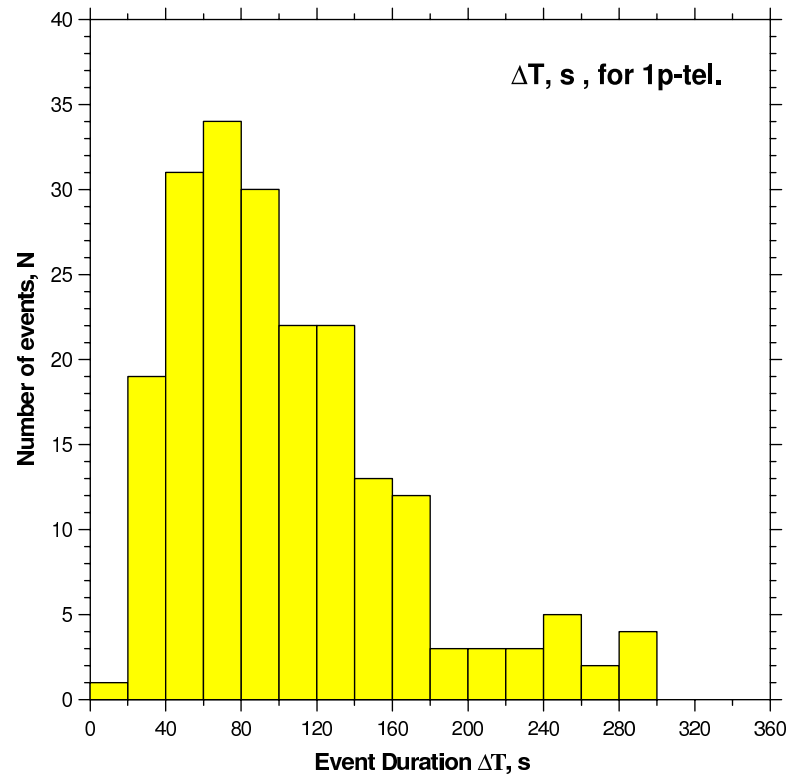

Fig. 5. The histogram of AMI event durations $\Delta T$.
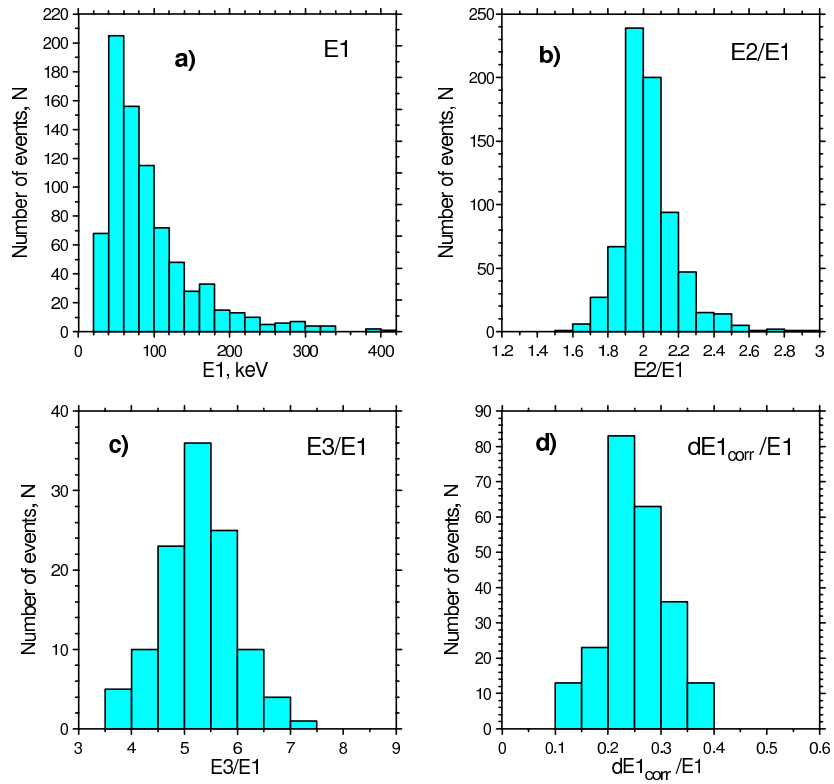

Fig. 6. Histograms for $E 1$ (a), $E 2 / E 1$ (b), $E 3 / E 1$ (c) and $d E 1_{\text {cor }} / E 1$ (d) (see text for details).

Figure 6 gives the histograms of values of the first line energy $E 1$, of line energies ratios $E 2 / E 1, E 3 / E 1$, and of relative values of the first line FWHM $d E 1_{\text {cor }} / E 1$. In the last case, from the measured value $d E 1$ the instrument resolution $d E 1_{\text {ins }}$ was excluded: $d E 1_{\text {cor }}=\sqrt{d E 1^{2}-d E 1_{\text {ins }}^{2}}$. The $d E 1_{\text {ins }}$ values were regularly measured during the in-flight instrument calibrations. The absolute intensity $\mathrm{J} 1$ in the maximum of the first line vary widely in different events, from 0.3 to $1.0 \times 10^{5} \mathrm{~cm}^{-2} \mathrm{~s}^{-1} \mathrm{sr}^{-1} \mathrm{keV}^{-1}$. 

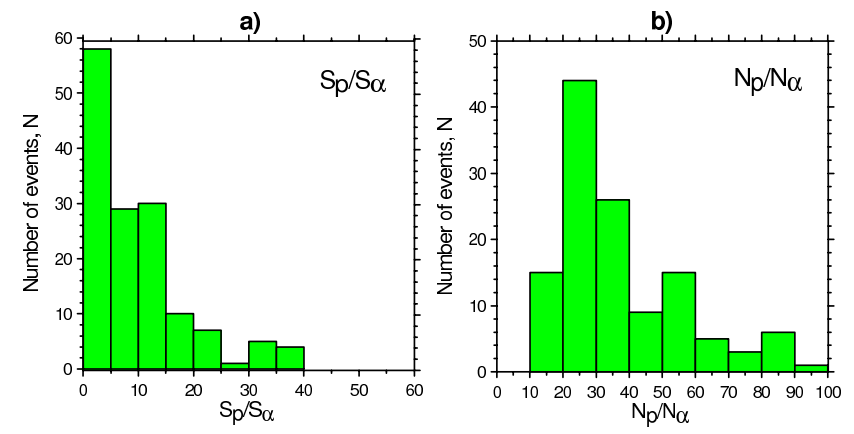

Fig. 7. (a) Histogram for the ratio of proton and alpha-particles line areas $\left(S_{\mathrm{p}} / S_{\alpha}\right)$ in AMI spectra. (b) Histogram for the ratio of protons and alpha-particles densities in the solar wind $\left(N_{\mathrm{p}} / N_{\alpha}\right)$.

Further in this section, we will denote the parameters of the first line (protons): energy and intensity at the maximum and corrected FWHM as $E_{\mathrm{p}}, J_{\mathrm{p}}$, and $d E_{\mathrm{p}}$ and similarly for the 2nd line (alpha-particles). Figure 7a shows the distribution of line areas ratio of protons $S_{\mathrm{p}}$ to alpha-particles $S_{\alpha}$ in the AMI spectra. Since the shapes of all the lines (Gaussians) hardly differ, we accept that

$\frac{S_{\mathrm{p}}}{S_{\alpha}}=\frac{J_{\mathrm{p}} \cdot d E_{\mathrm{p}}}{J_{\alpha} \cdot d E_{\alpha}}$,

Figure $7 \mathrm{~b}$ gives the distribution for ratio of densities protons and alpha particles $N_{\mathrm{p}} / N_{\alpha}$ in the solar wind plasma during the AMI measurements (data from the Wind $\mathrm{S} / \mathrm{C}$ ).

To compare these ratios let's calculate $S_{\mathrm{p}} / S_{\alpha}$ starting from the $N_{\mathrm{p}} / N_{\alpha}$ :

$\left(S_{\mathrm{p}} / S_{\alpha}\right)_{\text {calc }}=\frac{N_{\mathrm{p}} \cdot V_{\mathrm{p}} \cdot d E_{\mathrm{p}}}{N_{\alpha} \cdot V_{\alpha} \cdot d E_{\alpha}}$,

where $V_{\mathrm{p}} / V_{\alpha}$ is the ratio of ion velocities after the acceleration. As $E_{\mathrm{p}} / E_{\alpha}=0.5, d E_{\mathrm{p}} / d E_{\alpha}=0.5$, and $V_{\mathrm{p}} / V_{\alpha}=\sqrt{2}$, we obtain for the calculated value of $S_{\mathrm{p}} / S_{\alpha}$ :

$\left(S_{\mathrm{p}} / S_{\alpha}\right)_{\mathrm{calc}}=\frac{N_{\mathrm{p}} \cdot \sqrt{2}}{N_{\alpha} \cdot 2}=0.707 \cdot \frac{N_{\mathrm{p}}}{N_{\alpha}}$.

The average value for $N_{\mathrm{p}} / N_{\alpha}$ is equal to 37.26 (Table 2). Thus, for the ratio $\left(S_{\mathrm{p}} / S_{\alpha}\right)_{\text {calc }}$ we should have $\left(S_{\mathrm{p}} / S_{\alpha}\right)_{\text {calc }}=$ $37.26 \cdot 0.707=26.34$. In reality we have $S_{\mathrm{p}} / S_{\alpha}=12.35(\mathrm{Ta}-$ ble 2), i.e. it is 2.1 times lower. This difference is explained by our AMI acceleration model, which gives an advantage to alpha-particles over protons by monoenergetic lines creation (see Sect. 7).

\section{Angular distributions of AMI}

While DOK-2 telescopes could not examine the whole $4 \pi$ solid angle, in some cases we were able to estimate angular distributions of AMI and compare them with distributions of ions with usual, smooth spectra. As a first example, we

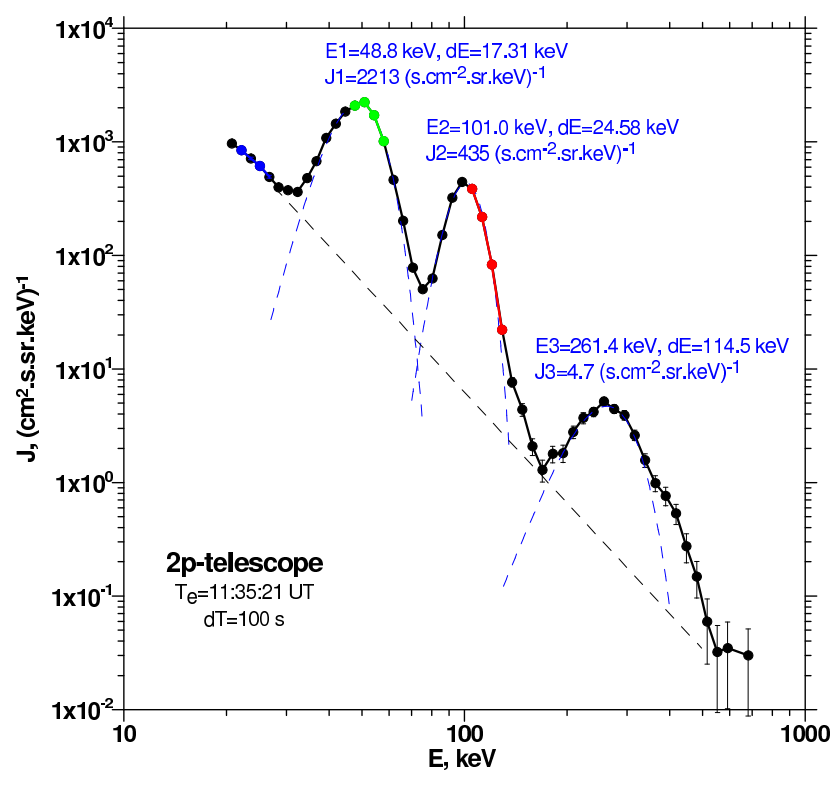

Fig. 8. The DOK-2 ion spectrum in the MSH on 23 April 1999, 11:33:21-11:35:21 UT.

present here results for the AMI event on 23 April 1999 in the $\mathrm{MSH}$, close to the BS. Figure 8 presents an ion spectrum from the 2 p-telescope, where 3 color intervals correspond to the 3 TP-parameters. One can see that TP1 is a part of a usual smooth background spectrum, while TP 2 and TP 3 correspond to the first two AMI lines. As mentioned in Sect. 2, time variations of TP-parameters were measured with a variable time resolution depending on variability of counts in 1-s BMs in the TP1 and TP3 energy intervals. Energy values for the TP intervals are shown in Fig. 9 (upper panel). When the variability was high, this resolution reached $1 \mathrm{~s}$. The $2 \mathrm{p}$ telescope with an angular aperture of $12.5^{\circ}$ was directed at the angle $62^{\circ}$ to the S/C spin axis pointed toward the Sun. By the $\mathrm{S} / \mathrm{C}$ rotation, the telescope scans the corresponding cone, which allows to estimate the angular distributions for AMI (TP2, TP3) and for a background spectrum (TP1).

Figure 9 shows time profiles for the three TP-parameters, as well as for magnetic field and angles of an ion flux unit vector in a local magnetic field (LMF) frame: a pitch angle (PA) and an azimuth angle (AA). LMF is a rectangular coordinate system in which $\boldsymbol{Z}_{\mathrm{LMF}}=\boldsymbol{B} /|\boldsymbol{B}|, \boldsymbol{Y}_{\mathrm{LMF}}=\left(\boldsymbol{Z}_{\mathrm{LMF}} \times\right.$ $\left.\boldsymbol{X}_{\mathrm{GSE}}\right)$, and $\boldsymbol{X}_{\mathrm{LMF}}=\left(\boldsymbol{Y}_{\mathrm{LMF}} \times \boldsymbol{Z}_{\mathrm{LMF}}\right)$. The pitch angle PA is the angle between the ion flux direction and the axis $Z_{\mathrm{LMF}}$, the azimuth angle (AA) corresponds to the rotation around the $Z_{\mathrm{LMF}}$ axis. All the data were averaged in time intervals of TP measurements. The time interval for the spectrum from Fig. 8 is shown in the upper panel. The vertical lines in Fig. 9 correspond to the times of maximum intensity of AMI (solid lines) and of ions with the usual spectrum (dashed lines). The values of pitch and azimuth angles for these times are shown in the lower frame. Time intervals between these maxima correspond to $\sim 2 \mathrm{~min}$ spin period of the S/C. The intensity 

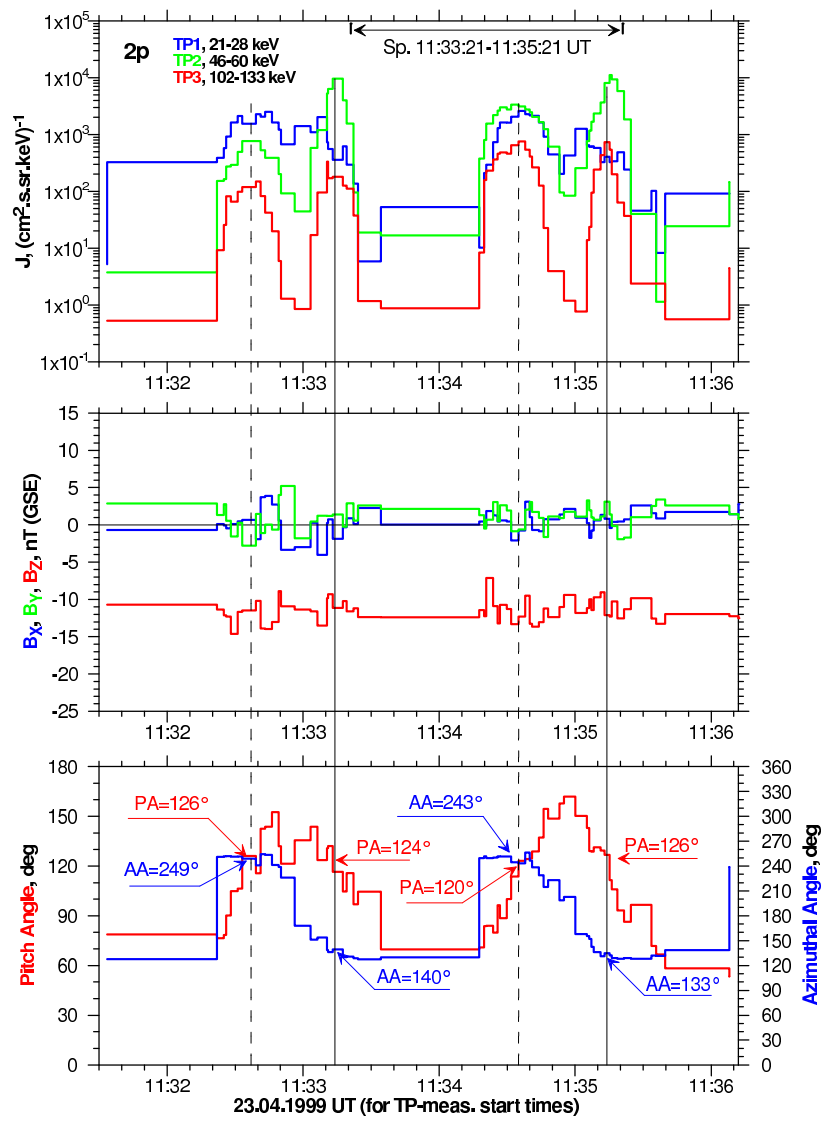

Fig. 9. Time variations of TP-parameters, magnetic field, and ion flux angles in LMF frame for the AMI event in the MSH near the Bow Shock on 23 April 1999 (see text for details).

peaks for AMI are narrower than for the usual spectrum. A more detailed analysis showed that the FWHM of these peaks for AMI corresponds to intervals of PA and AA $<20^{\circ}$. Since this value also includes the telescope aperture $\left(12.5^{\circ}\right)$, the intrinsic FWHM of the AMI beam must be no more than $10-15^{\circ}$.

Figure 10 presents a summary of relative angular distributions for 3 TP-parameters in the whole considered time interval. One can see that the distribution for the usual spectrum (upper diagram) is broader and the azimuth angles for the intensity maxima differ strongly for AMI $\left(133^{\circ}\right.$ to $\left.140^{\circ}\right)$ and for the usual spectrum $\left(243^{\circ}\right.$ to $\left.249^{\circ}\right)$.

In Figs. 11-13, one more example of AMI observation is presented, which took place on 16 April 1996 at 18:35:4318:45:35 UT in the MSH close to the BS. In Fig. 11 as well as in Fig. 8, three colors mark energy intervals corresponding to the three TP-parameters. Here the first lines of AMI (with the exception of the spectrum $\sharp 3$ ) fall into the energy range of the TP3. It also allowed the comparison of angular distributions for AMI and for ions with the usual smooth spectrum (TP1, TP2). Periodical (2 min) increases of ion intensities (Fig. 12, upper panel), as in the previous case, correspond to the spin
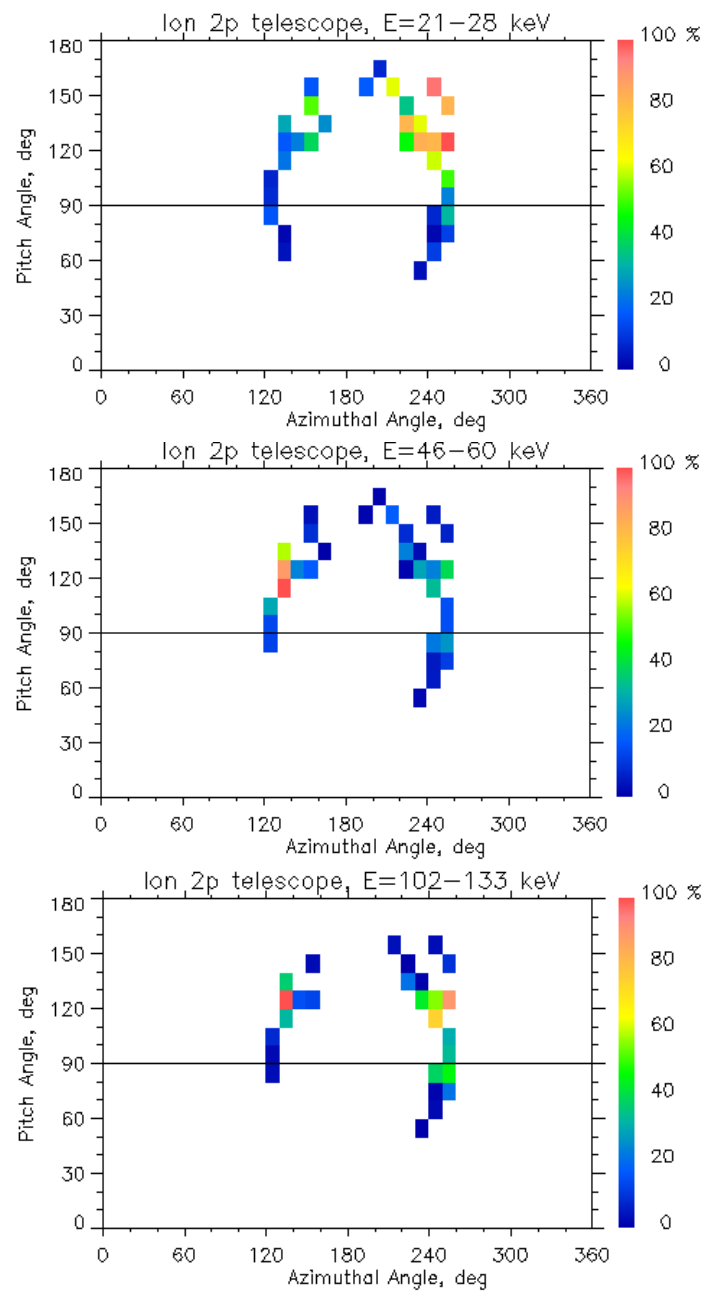

Fig. 10. Angular distributions for the AMI event in the MSH on 23 April 1999 at 11:31:00-11:37:00 UT.

period. In the upper panel the time intervals for spectra $\sharp 2$ $\sharp 7$ are shown. Thin vertical lines in Fig. 12 correspond to the flux maxima of ions with a usual spectrum (TP1, TP2, dashed lines) and for AMI (TP3, solid lines). They allow to find the pitch and azimuth angles for these moments (see bottom panel). The maxima of TP3 (AMI) fall in the pitch angles from $63^{\circ}$ to $66^{\circ}$ and in two ranges of the azimuth angles: $133^{\circ}$ to $140^{\circ}$ and $227^{\circ}$ to $234^{\circ}$. The maxima for TP1 and TP2 (usual spectrum) fall at the pitch angles $107^{\circ}$ to $116^{\circ}$ and azimuth angles from $247^{\circ}$ to $248^{\circ}$. The difference in these angles for both types of spectra is illustrated by the diagrams in Fig. 13.

It follows from the discussed examples that the angular distributions for AMI beams are narrow $\left(\mathrm{FWHM}<15^{\circ}\right.$ ) and differ strongly from the distributions for ions with usual spectra. These facts point to small dimensions of the acceleration region and short duration of the process. 

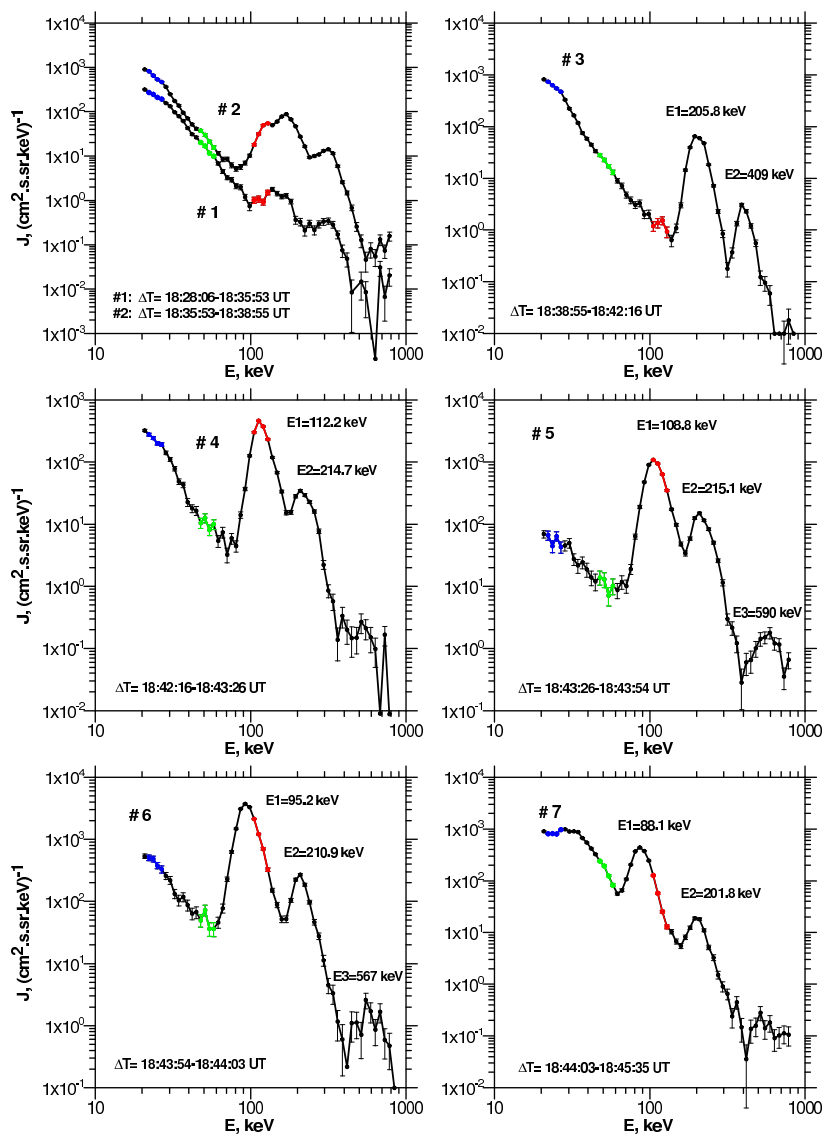

Fig. 11. AMI spectra (2p-telescope) in the MSH near the BS on 16 April 1996 at 18:35:53-18:45:35 UT

\section{The change of energies and intensities of proton lines after the AMI event start}

For the AMI events with high time resolution in spectra measurements ( 2 to $10 \mathrm{~s}$ ), we have plotted and analyzed the time dependence of energies and intensities of proton lines after the start of the acceleration process. The results for 6 such AMI events are given in Fig. 14. Evidently, the proton line energy E1 consists of two parts: a constant one (A) and a second one (B) exponentially decreasing with time. In a frame of our hypothesis, the first one, obviously, corresponds to the electric circuit electromotive force (EMF), the second one to the self-inductance EMF, arising after the circuit disruption and depending on the circuit inductance (length). The time $\mathrm{T} 0$ corresponds to the events start $(\mathrm{J} 1 \approx 0)$. The time dependence of E1 was approximated by the expression:

$E 1=A+B \cdot \exp (-C \cdot(T-T 0))$

The values obtained for the parameters $A, B, C$, and $T 0$ are given in Table 3. A very fast increase of intensities J1 (blue points and lines in Fig. 14) immediately after the current disruption can be explained by a fast filling of an almost "empty" disruption region by the MSH plasma (see Sect. 8).



Fig. 12. Time variations of TP-parameters, magnetic field and angles of ion fluxes in LMF-frame for AMI event on 16 April 1996.

A smooth decrease of the proton line energy here cannot be a result of the time-of-flight dispersion during the propagation of particles with the usual spectra from the site of their acceleration to the points of registration. Firstly, in this case, the energy $E$ change with time $T$ would have happened under the law: $E=M \cdot S^{2} / 2 /\left(T-T_{\mathrm{g}}\right)^{2}$, where $M$ is the mass of the particle, $S$ is the distance to the source, and $T_{\mathrm{g}}$ is the time of particles generation. This dependence, in contrast to the Eq. (2), has no constant component and has a quite different shape. Secondly, the energy ratio of alpha-particles and protons would have been equal to 4 , not 2 as in our observations. Of course, our spectra were measured not right after the acceleration, but with a delay depending on time of flight of protons to the observation point. Rough estimate of these delays for the 6 events showed that they ranged from 1.5 to $24 \mathrm{~s}$, which cannot substantially change the results of our analysis.

\section{Model of AMI acceleration}

Previously on the basis of our preliminary analysis of AMI events we proposed a hypothesis (Lutsenko and Kudela, 
Table 3. Parameters of the time dependence of the first line energy E1 near the acceleration start for six AMI events (see Fig. 14). T0 is in seconds of the day.

\begin{tabular}{lcccccc}
\hline Date & UT interval & Tel. & $\begin{array}{c}\text { T0, } \\
\mathrm{s}\end{array}$ & $\begin{array}{c}\mathrm{A}, \\
\mathrm{keV}\end{array}$ & $\begin{array}{c}\mathrm{B}, \\
\mathrm{keV}\end{array}$ & $\begin{array}{c}\mathrm{C}, \\
1 / \mathrm{s}\end{array}$ \\
\hline 6 Jun 1996 & $12: 24: 30-12: 25: 00$ & $1 \mathrm{p}$ & 44672 & 26.65 & 34.82 & 0.082 \\
9 Apr 2000 & $03: 08: 00-03: 10: 00$ & $1 \mathrm{p}$ & 11290 & 55.84 & 32.89 & 0.058 \\
12 Apr 1996 & $22: 22: 20-22: 23: 20$ & $2 \mathrm{p}$ & 80552 & 22.5 & 29.74 & 0.04 \\
10 Aug 1997 & $23: 13: 35-23: 14: 54$ & $1 \mathrm{p}$ & 83626 & 46.2 & 45.3 & 0.035 \\
23 Apr 1997 & $20: 38: 30-20: 39: 45$ & $1 \mathrm{p}$ & 74319 & 48.9 & 22.8 & 0.06 \\
20 Apr 1999 & $13: 43: 20-13: 44: 25$ & $1 \mathrm{p}$ & 49402 & 80.43 & 59.47 & 0.09 \\
\hline
\end{tabular}
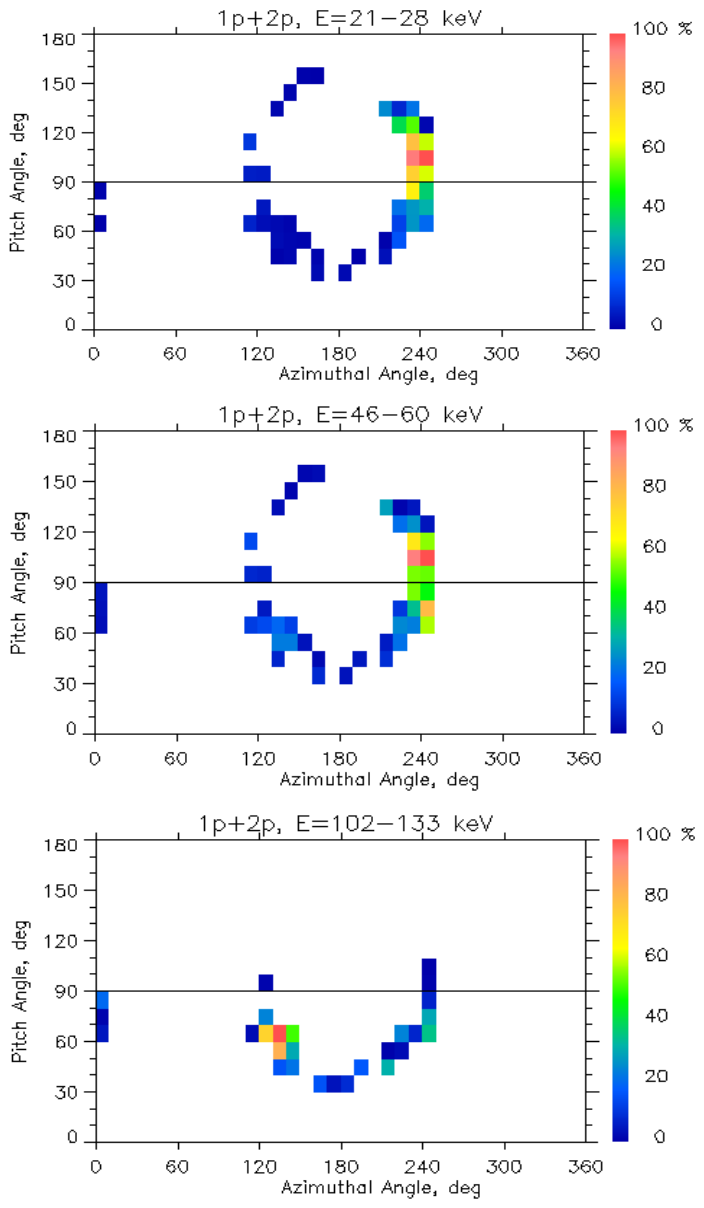

Fig. 13. Angular distributions for the AMI event in the MSH on 16 April 1996 at 18:36:12-18:44:33 UT.

1999; Lutsenko, 2001) in which generation of AMI observed near BS occurred in spikes of potential electric field arising as a result of BS current sheet filaments disruptions (see Fig. 15). Like each current circuit, these filaments have an electromotive force EMF ( $A$ in Fig. 15), a resistance $R$, and an inductance $L$. The EMF is distributed along that part of the circuit which is exposed to an action of a motional elec- tric field of the SW. After the circuit disruption, a sum of the EMF and the self-inductance EMF ( $B$ in Fig. 15) will be applied to the short disruption interval $W$ as a voltage difference $\Delta V=A+B$.

This assumption was entirely confirmed by the results of our full analysis of the $\sim 730$ AMI events, in particular:

1. by the positions of AMI observation points,

2. by the energy ratios of AMI lines corresponding to the ratios of $\mathrm{H}, \mathrm{He}$, and the $\mathrm{CNO}$-group charges,

3. by the average energy of the proton line (see Table 2), which is not inconsistent with the average potential drop across the magnetosphere due to the motional electric field of the solar wind: $\boldsymbol{E}_{\mathrm{sw}}=-\left(\boldsymbol{V}_{\mathrm{sw}} \times \boldsymbol{B}_{\mathrm{sw}}\right)$,

4. by short durations of AMI events (on average 104.7 s),

5. by the character of time variations of proton line energies and intensities at the event start. It is this character of voltage $\Delta V$ change that can be expected by the disruption of the electric circuit, shown in Fig. 15, and

6. by the total absence of monoenergetic electrons in the AMI events.

Let's examine now in more detail the acceleration process and limitations on dimensions and other characteristics of the acceleration region resulting from our model. Let us assume that the Y-axis is directed along the current sheet filament (along the potential electric field $\boldsymbol{E}$ ), the $\mathrm{X}$-axis is perpendicular to the filament, and the Z-axis is directed along the magnetic field. An ion trajectory in the crossed $\boldsymbol{E}$ and $\boldsymbol{B}$ fields $(\boldsymbol{E} \perp \boldsymbol{B})$ is a cycloid, and acceleration of ions up to the same energy level will be possible if the cycloid width $Y_{\max }$ in the Y-direction is notably larger than the acceleration region dimension $W$ in this direction. Let's find the resulting limitations for the $W$-value. The equation of ions motion in the crossed electric $\boldsymbol{E}$ and magnetic $\boldsymbol{B}$ fields:

$M \cdot \frac{d \boldsymbol{v}}{d t}=\frac{Z \cdot e}{c}(\boldsymbol{v} \times \boldsymbol{B})+Z \cdot e \cdot \boldsymbol{E}$ 

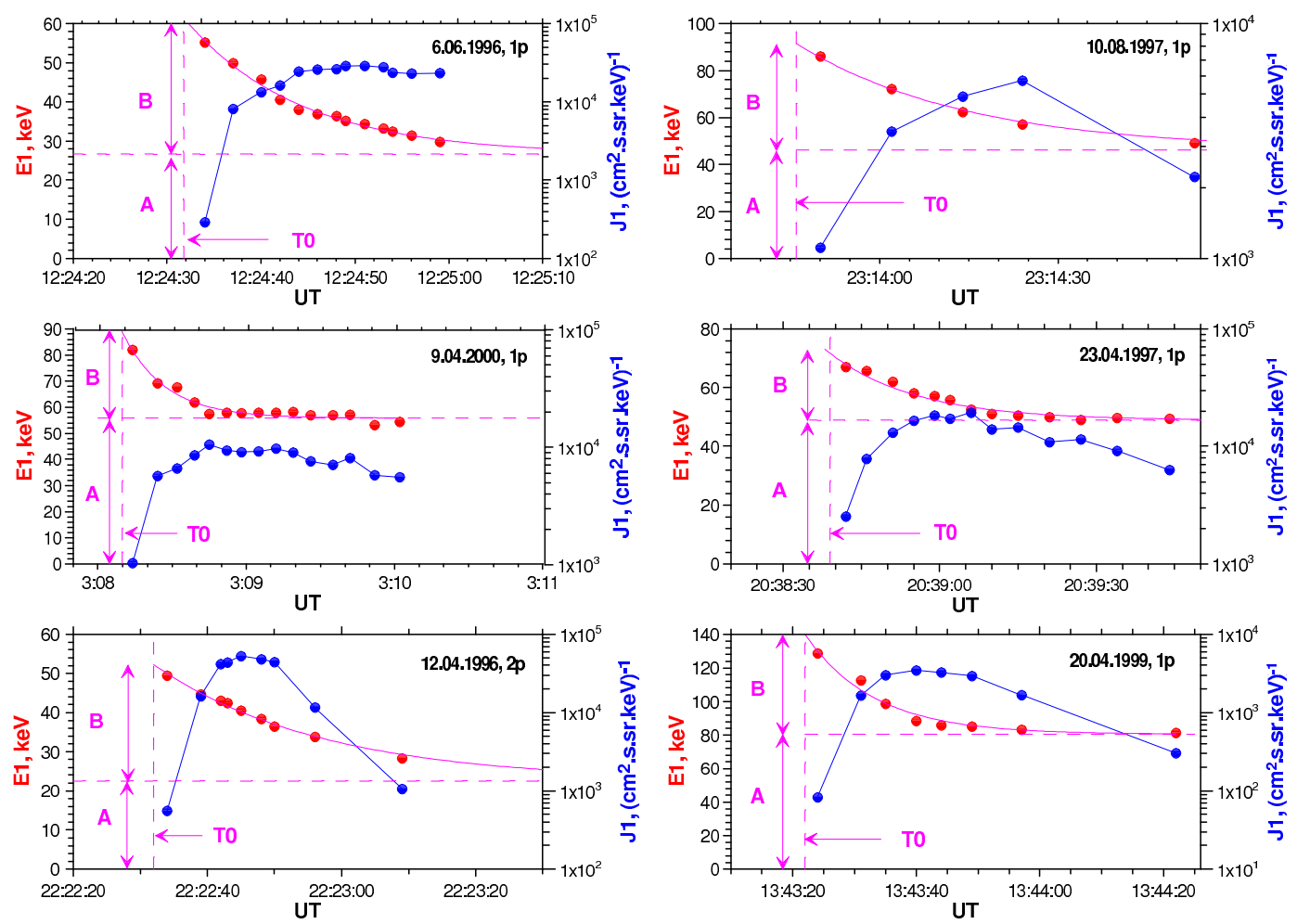

Fig. 14. Time variations of energies $E 1$ and intensities $J 1$ of AMI proton lines near the events start.

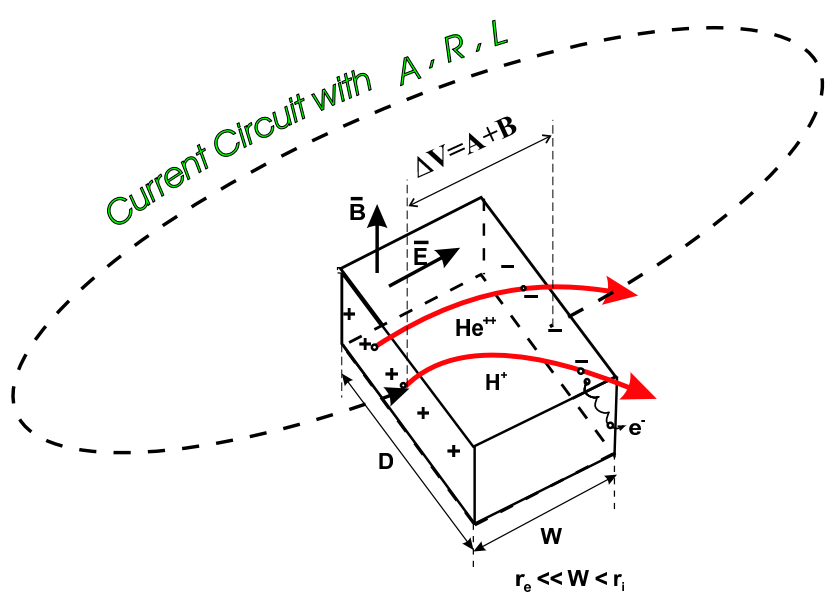

Fig. 15. The sketch illustrates the process of solar wind ions acceleration and AMI generation by BS current sheet filaments disruption. Here, $A$ is the circuit electromotive force (EMF), $B$ is the self-inductance EMF. Electrons, having much lower gyroradia, will be swept out by the drift without the acceleration.

With $E_{\mathrm{y}}=E, \quad E_{\mathrm{x}}=E_{\mathrm{z}}=0, \quad B_{\mathrm{x}}=B_{\mathrm{y}}=0, \quad B_{\mathrm{z}}=B$ it gives:

$\frac{d v_{\mathrm{x}}}{d t}=\omega_{\mathrm{c}} \cdot v_{\mathrm{y}}, \quad \frac{d v_{\mathrm{y}}}{d t}=-\omega_{\mathrm{c}} \cdot v_{\mathrm{x}}+\frac{Z \cdot e \cdot E}{M}$
Here: $\omega_{\mathrm{c}}$ is the cyclotron frequency, $\omega_{\mathrm{c}}=(Z \cdot e \cdot B) / M=$ $9.579 \times 10^{-2} \cdot Z \cdot B / A$ in $\operatorname{rads}^{-1}, Z \cdot e$ is the ion charge in $\mathrm{C}$, $M=A \cdot m_{\mathrm{p}}$ is the ion mass in $\mathrm{kg}, B$ is the magnetic induction in $\mathrm{nT}$, the ratio $e / m_{\mathrm{p}}=9.579 \times 10^{7} \mathrm{C} \mathrm{kg}^{-1}$. The solution of Eq. (3) for an initial velocity $\bar{v}_{0}=0$ :

$x=\frac{Z \cdot e \cdot E}{M \cdot \omega_{\mathrm{c}}} \cdot t-\frac{Z \cdot e \cdot E}{M \cdot \omega_{\mathrm{c}}^{2}} \cdot \sin \left(\omega_{\mathrm{c}} \cdot t\right)$,

$y=\frac{Z \cdot e \cdot E}{M \cdot \omega_{\mathrm{c}}^{2}} \cdot\left(1-\cos \left(\omega_{\mathrm{c}} \cdot t\right)\right)$

or denoting $K=1.044 \times 10^{7} \cdot(A \cdot E) /\left(Z \cdot B^{2}\right)$, in $\mathrm{km}$ :

$x=K \cdot\left(\omega_{\mathrm{c}} \cdot t-\sin \left(\omega_{\mathrm{c}} t\right)\right), \quad y=K \cdot\left(1-\sin \left(\omega_{\mathrm{c}} t\right)\right)$,

also in $\mathrm{km}$. The cycloid width is:

$Y_{\max }=\frac{2 \cdot Z \cdot e \cdot E}{A \cdot m_{\mathrm{p}} \cdot \omega_{\mathrm{c}}^{2}}=2.088 \times 10^{7} \cdot \frac{A}{Z} \cdot \frac{E}{B^{2}}, \quad \mathrm{~km}$

The values of the $\omega_{\mathrm{c}}$ and $Y_{\max }$ for $B=10$ and $20 \mathrm{nT}$ and $E=0.1 \mathrm{~V} \mathrm{~m}^{-1}$ are given in Table 4 .

So for the acceleration of all protons up to the same energy level, the width of the acceleration area $W$ in the direction of electric field must be $\ll 5000 \mathrm{~km}$ at $B=20 \mathrm{nT}$ and $\ll 20000 \mathrm{~km}$ at $B=10 \mathrm{nT}$. For heavy ions this limitation is not so strong. Figure 16a shows proton trajectories in the acceleration region with the thickness $D$ (the current filament 
Table 4. Cyclotron frequency and $Y_{\max }$ for different ions by $E=0.1 \mathrm{~V} \mathrm{~m}^{-1}$.

\begin{tabular}{ccccccccc}
\hline & & \multicolumn{3}{c}{$B=20 \mathrm{nT}$} & & \multicolumn{3}{c}{$B=10 \mathrm{nT}$} \\
\cline { 3 - 5 } \cline { 6 - 8 }$Z n$ & $A$ & $\omega_{\mathrm{c}}, \mathrm{rad} \mathrm{s}^{-1}$ & $Y_{\max }, \mathrm{km}$ & $Y_{\max }, R_{\mathrm{E}}$ & & $\omega_{\mathrm{c}}, \mathrm{rad} \mathrm{s}^{-1}$ & $Y_{\max }, \mathrm{km}$ & $Y_{\max }, R_{\mathrm{E}}$ \\
\hline 1 & 1 & 1.916 & 5217.5 & 0.818 & & 0.958 & 20870 & 3.272 \\
2 & 4 & 0.958 & 10435 & 1.636 & & 0.479 & 41740 & 6.544 \\
6 & 16 & 0.719 & 13913 & 2.181 & & 0.359 & 55652 & 8.726 \\
\hline
\end{tabular}

thickness) and disruption region width $W$. If $W>Y_{\max }$, the creation of monoenergetic lines is impossible. One can see that all protons, starting within the limits of $D_{\text {eff }}$, will obtain the same final energy of $200 \mathrm{keV}$. Protons, starting out

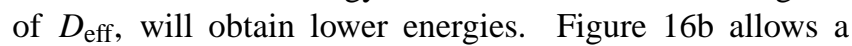
comparison of protons and alpha-particles acceleration. It follows from this figure that the effective value $D_{\text {eff }}$ is always greater for alpha-particles than for protons. Indeed, for $W=4000 \mathrm{~km} D_{\text {eff }}^{\alpha} / D_{\text {eff }}^{\mathrm{p}}=1.31$ and for $W^{\prime}=5000 \mathrm{~km}$ (dashed lines), the mentioned ratio will be equal to 2.65. So we must expect that after leaving of the acceleration region, alpha-particles will always have an advantage over protons at creation of monoenergetic lines. It explains why the value of ratio $\left(S_{\mathrm{p}} / S_{\alpha}\right)_{\text {calc }}$ calculated from the ratio $N_{\mathrm{p}} / N_{\alpha}$ is $\sim 2$ times lower than the observed average value of $S_{\mathrm{p}} / S_{\alpha}$ (see Sect. 4). Graphics in Fig. 16 and data in Table 4 allow us to make rough estimates of the acceleration region size. The width of the accelerating gap $W$ should not exceed $Y_{\max }=0.818 R_{\mathrm{E}}$ for $B=20 \mathrm{nT}$. The thickness of the acceleration region $D$ can not be greater than $1.25 R_{\mathrm{E}}$ for a conservation of the observed advantage of alpha particles over protons in a factor of $\sim 2$ by monoenergetic lines formation.

\section{The reason of current circuit disruption - connection between AMI and HFA}

The natural question arises: what is the reason of current filaments disruptions? In the magnetotail neutral sheet where AMI were also observed, the disruptions of filaments of the current sheet separating oppositely directed magnetic fields may be spontaneous. Their durations amount to $\sim 20 \mathrm{~s}$, after which the conductivity is restored (see Figs. 14 and 15 from Lutsenko et al., 2008). In the case of BS, the current sheet separates magnetic fields of about the same directions so one should look for an external reason of disruptions.

In the solar wind near BS, a phenomenon known as Hot Flow Anomaly (HFA) (see e.g. Schwartz, 1995; Schwartz et al., 2000) was often observed. HFA was observed when the solar wind had brought to the BS a tangential discontinuity in the magnetic field caused by a large-scale flat current sheet (CS). Because of opposite directions of the magnetic fields on both sides of the CS, the convection electric fields near the CS-BS intersection line may be directed to the CS. This electric field changes the plasma flow direction, so that the solar
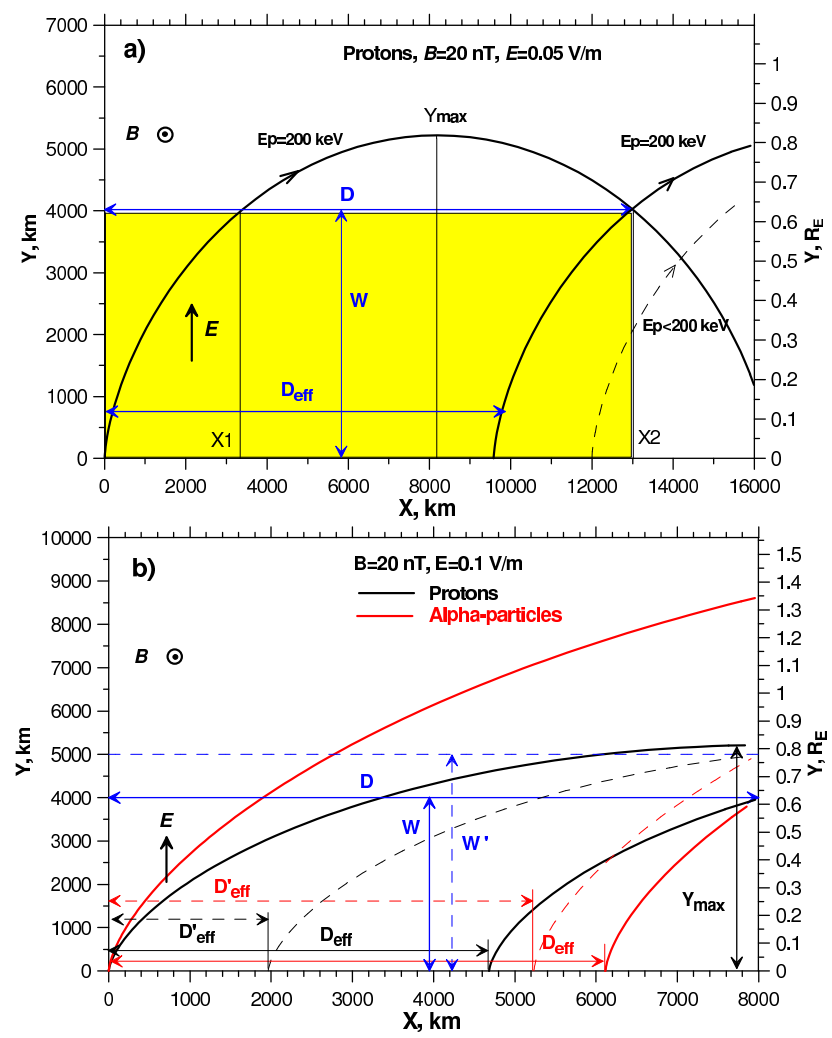

Fig. 16. (a) Proton trajectories in the acceleration region. $D-$ the thickness of the disrupted current filament. $W-$ the width of the region with the potential electric field. $D_{\text {eff }}$ - effective thickness of the acceleration region, starting from which all protons will obtain the same energy $200 \mathrm{keV}$. Protons, starting out of $D_{\text {eff }}$ limits, will obtain the energy $<200 \mathrm{keV}$. (b) Comparison of $D_{\text {eff }}$ for alphaparticles and for protons. Dotted lines show the same trajectories for the larger value of the acceleration region width $W^{\prime}=5000 \mathrm{~km}$ instead of $W=4000 \mathrm{~km}$.

wind flux deflects through a large angle to the CS position and upon reaching the CS it even turns sunward (Schwartz, 1995). In these studies, the influence of HFA on such a largescale BS parameter as its current sheet was not considered.

In Fig. 17 we would like to explain the physical nature of BS current sheet and to show a possible reason for its filaments disruption. Figure 17a illustrates the creation of current carriers (ions and electrons) on a BS magnetic field 
a)

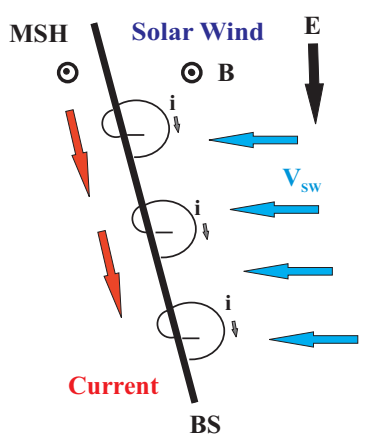

b)

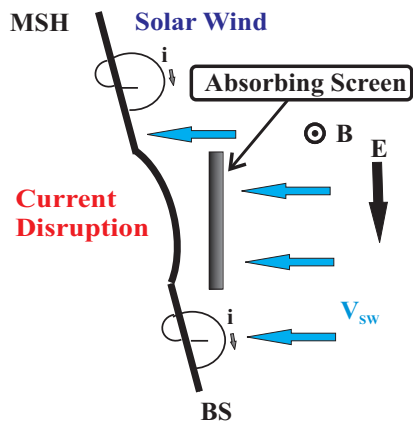

c)

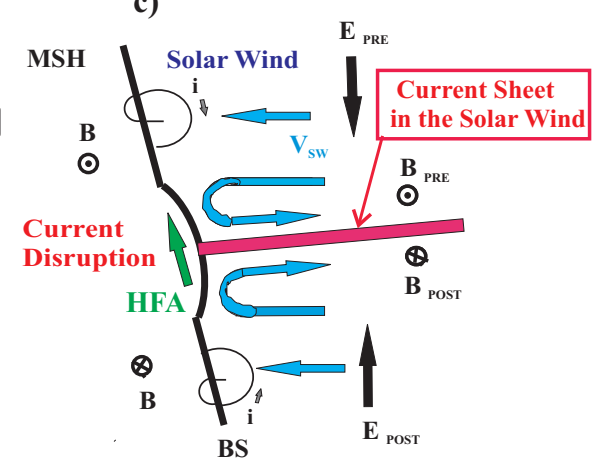

Fig. 17. The HFA phenomenon as a possible cause for BS current sheet filaments disruptions. (a) Creation of current carriers for the BS current sheet. (b) How the BS current sheet can be disrupted. (c) How the HFA can play a role in the screen in Fig. 17b.

jump. Solar wind ions and electrons crossing this jump perform a short gradient drift, shifting them along a tangential component of the motional electric field of the solar wind. Thus, they serve as charge carriers for the BS current. Then they move downstream to the MSH, being replaced by new SW particles. Let us suppose that we have set somewhere upstream of the BS an absorbing screen protecting a part of BS from the solar wind flux (Fig. 17b). Creation of current carriers here stops and the BS current sheet or, better said, a part of its filaments, becomes disrupted. Figure 17c shows how the arrival of CS in the solar wind, creation of the HFA, and deviation of plasma flow lead to a situation with disruption of the BS current similar to the screen action in Fig. 17b. An observer situated at a small distance behind the BS will see a decrease of the antisunward plasma flux to about a zero level, while the fluxes perpendicular to the sun direction must increase.

After the solar wind shifts the line of CS and BS intersection further (in the direction of the green arrow in Fig. 17c), the antisunward plasma flux will not only be restored but can become more intense due to addition of the solar wind plasma deflected and detained by HFA (see, e.g. Sect. 9.2, Fig. 23). The disruption disappears and can arise in the neighboring filaments.

We have paid attention to a similarity of conditions for generation of AMI and creation of HFA:

1. Arrival of the CS in the solar wind,

2. The CS is almost perpendicular to BS at times of AMI and HFA generation (the angle between normals $N_{\text {cs }}$ and $\boldsymbol{N}_{\mathrm{bs}}$ is close to $90^{\circ}$ ),

3. Quasi-perpendicular orientation of the magnetic field (the angle $T_{\mathrm{bn}}$ is close to $90^{\circ}$ ) at least on one side of CS (to reflect ions), and

4. The electric field at least at one CS side is directed to the CS.
The rate of HFA occurrence $(\sim 0.12$ per hour $)$ given by Schwartz et al. (2000) is consistent with our estimates of AMI events occurrence rates ( $>0.038$ per hour). Our analysis of the AMI events and presence of CS in the solar wind has shown that:

$\sim 40 \%$ of AMI events were accompanied by a strong, single CS,

$\sim 30 \%$ events - by weak or numerous CSs - what complicates the analysis,

$\sim 30 \%$ events - by the absence of CS - that points to the possibility of other reasons for current disruptions.

However, these general considerations should be supported by direct observations of CS, HFA, and AMI in one particular event. For such analysis it is necessary to select observations that occurred in the solar wind or in the magnetosheath close enough ( 1 to $2 R_{\mathrm{E}}$ ) to the BS. Otherwise, it will be difficult to detect AMI and HFA on the same S/C. We must also bear in mind that AMI propagate from the acceleration place as a narrow beam and can not always be detected on the $\mathrm{S} / \mathrm{C}$, even if its position is close to the acceleration place. On the other hand, depending on the direction of magnetic field connecting the acceleration place with the S/C, the AMI observation time can be as before, as after the moment of the CS and HFA observation (delay of 1 to $10 \mathrm{~min}$ ). In such cases, AMI can be connected with the other HFA generated by the same CS several minutes before or after in a neighboring place.

\section{Examples of several AMI events analysis}

To analyze the AMI events and check fulfillment of the 4 conditions specified in the previous section, it is necessary to find a CS which can be responsible for generation of HFA and AMI using magnetic field data from one or several S/C in the solar wind. Then it is necessary to determine the CS parameters allowing a modeling of its motion by intersection with the BS (a direction of CS normal $N_{\mathrm{cs}}$ and velocity 
$V_{\text {csn }}$ of its motion along this normal). The minimum variance analysis of the magnetic field near the CS allows finding the normal to the current sheet $\boldsymbol{N}_{\text {cs }}$. Then the normal component of the CS velocity $V_{\text {csn }}$ can be found by using $N_{\mathbf{c s}}$ and measured solar wind velocity $\boldsymbol{V}_{\mathbf{s w}}$. Among the two possible $N_{\text {cs }}$ directions, the direction with $N_{\text {csx }}>0$ has always been selected. In that case for non-fulfillment of the 4-th condition, it is necessary that simultaneously $E_{\mathrm{nb}}<0$ and $E_{\mathrm{na}}>0$. Here, $E_{\mathrm{nb}}$ and $E_{\mathrm{na}}$ are the projections of the solar wind electric field on $N_{\mathrm{cs}}$ before and after CS coming, respectively. In all other cases the condition 4 is fulfilled. Below we give several examples of almost simultaneous observation of the CS, HFA, and AMI on Interball-1. The AMI observations were made by the DOK-2 instrument. The presence of HFA (the solar wind flux direction change) was identified by the VDP instrument (Safrankova et al., 1997), with $15 \mathrm{~s}$ time resolution. VDP had 4 ion detectors (Faraday cups). One (F1) was mounted to look sunward along the $\mathrm{S} / \mathrm{C}$ spin axis and three other (F2, F3, and F5) were mounted to look perpendicular to the spin axis with $90^{\circ}$ and $180^{\circ}$ azimuthal intervals. As Interball-1 had no direct electric field measurements, the $\boldsymbol{E}_{\mathrm{SW}}$ was calculated by using Wind measurements of $\boldsymbol{V}_{\mathrm{SW}}$ and $\boldsymbol{B}_{\mathrm{SW}}$ often made far from the HFA and AMI generation position. It may explain why in some cases below, the 4-th condition was not fulfilled.

\subsection{AMI events on 23 April 1997 in the solar wind near the BS}

Figure 18 presents positions of Interball-1 and Geotail during the observations on Interball-1 of two AMI events (AMI-1 and AMI-2), the spectra of which are shown in Fig. 19. The times of AMI-1 and AMI-2 observations are marked on the third panel of Fig. 20, where magnetic fields (Wind, Geotail and Interball-1) and solar wind fluxes (Interball-1) are presented.

Here we have two strong current sheets in the solar wind CS1 and CS2 which can be responsible for AMI-1 and AMI2. Interball-1 approached to the model BS by $0.65 R_{\mathrm{E}}$ between CS1 and CS2, what can explain the absence of obvious signs of HFA near the CS1 and their presence near the CS2. A double jump in magnetic field and plasma flux near the CS2 on Interball-1 may be caused by short oscillations of the BS position. Figures 21 and 22 present the positions of CS1 and CS2 at observations of AMI1 and AMI-2, as well as HFA (only for AMI-2). For the AMI-2 event, all 4 requirements are fulfilled $\left(E_{\mathrm{nb}}=\right.$ $\left.2.21 \mathrm{mV} \mathrm{m}^{-1}, E_{\mathrm{na}}=-1.36 \mathrm{mV} \mathrm{m}^{-1}\right)$. For AMI-1, all the requirements are fulfilled apart from the 4-th requirement $\left(E_{\mathrm{nb}}=-1.25 \mathrm{mV} \mathrm{m}^{-1}, E_{\mathrm{na}}=1.89 \mathrm{mV} \mathrm{m}^{-1}\right)$.

\subsection{AMI event on 16 April 1996 in the MSH near the BS}

On 16 April 1996, Interball-1 and Geotail were both in the MSH near the BS (see Fig. 2) observing an AMI event in
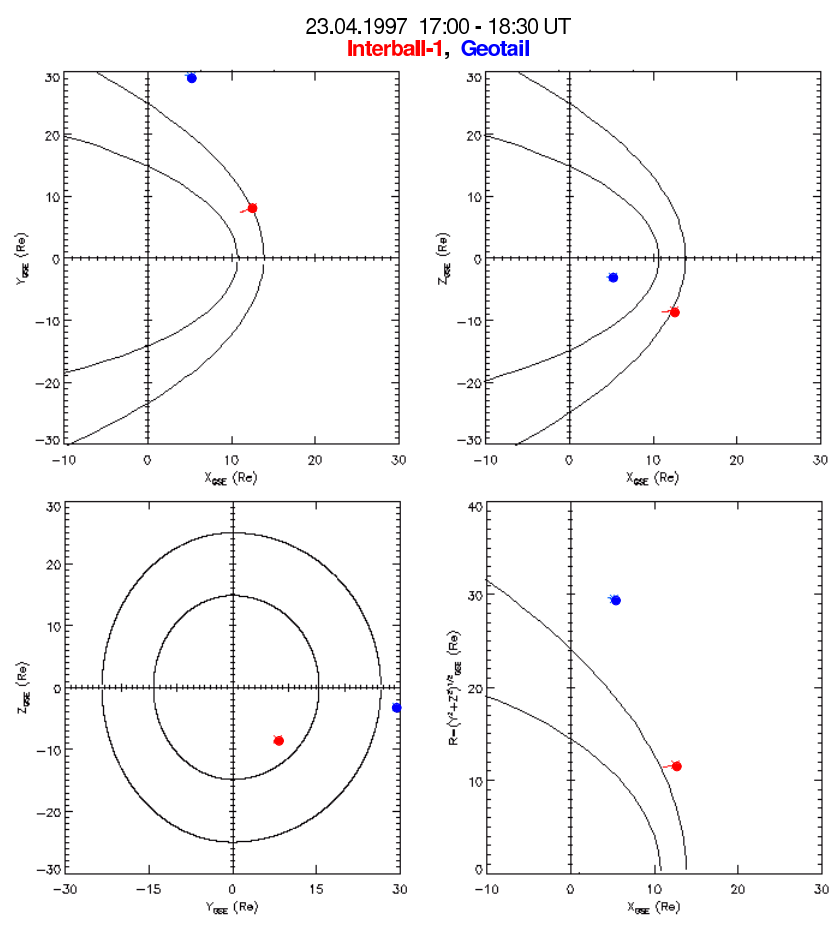

Fig. 18. Positions of Interball-1 and Geotail during AMI events on 23 April 1997.

time interval from 18:43 to 18:49 UT. The spectra of DOK-2 (2p-telescope) and the EPIC device for this event were also shown above (see Figs. 3 and 11). Figure 23 presents the magnetic fields for $3 \mathrm{~S} / \mathrm{C}$ and plasma fluxes (according to the VDP data). Times of AMI observations on S/C Interball-1 and Geotail are shown by short magenta lines in corresponding panels. The HFA is clearly seen here in the bottom panel in time interval from 18:47:29 to 18:50:52 UT as a drop of F1 flux to about zero and increase of F2, F3, and F5 fluxes. As both of these $\mathrm{S} / \mathrm{C}$ were in the $\mathrm{MSH}$ rather far from the model BS ( -2.6 and $\left.-1.42 R_{\mathrm{E}}\right)$, the CS6 distinctly visible in the Wind magnetic field is not seen in Interball-1 and Geotail magnetic field data. Nevertheless, we have shown that just the current sheet CS6 can be responsible for AMI generation. The results of this tangential discontinuity analysis and calculated position of the CS6 at the moments of HFA and AMI observations are given in Fig. 24. It is seen from this figure that the first 3 requirements are fulfilled. The electric field on one side of the CS6 (after the current sheet) is directed to the CS6: $E_{\mathrm{nb}}=-1.50, E_{\mathrm{na}}=-0.44 \mathrm{mV} \mathrm{m}^{-1}$, i.e. all 4 requirements are fulfilled.

\section{Conclusions}

1. The study of the large data array on AMI beams observed by Interball-1 near the BS showed that AMI beams are a fairly widespread phenomenon. The 

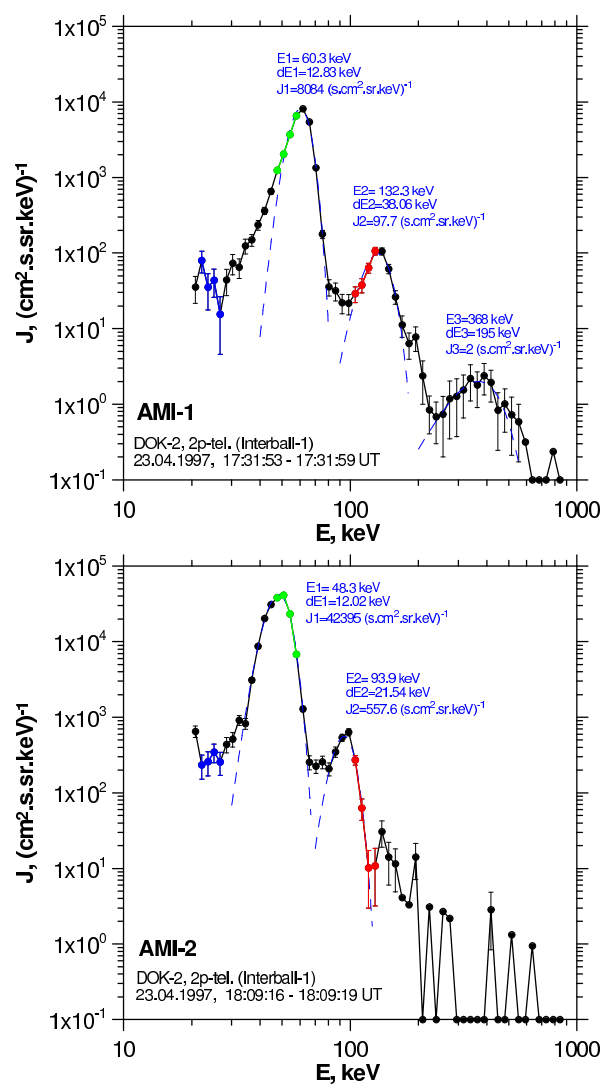

Fig. 19. Spectra of two AMI events on 23 April 1997.

average observation frequency in SW and MSH during the 5-year work of Interball-1 was 0.038 per hour.

2. The statistics of AMI properties based on a greater number of observations as well as the comparison of the DOK-2 data with the data from some other experiments confirm our assumptions on the nature of AMI lines and mechanism of their formation.

3. The formation of AMI beams is a result of the solar wind ions acceleration in small regions on the BS with a potential electric field created by disruptions of BS current sheet filaments. Such acceleration mechanism was confirmed, in particular:

- by the dynamics of energies and intensities of proton lines after the event start,

- by complete absence of monoenergetic electrons in the $25-400 \mathrm{keV}$ energy range during the AMI events,

- by ratios of energies of the three AMI lines attributed to $\mathrm{H}, \mathrm{He}$, and (CNO)-group, and

- by the marked difference between the ratios of numbers of accelerated protons and alpha-particles in AMI spectra and the same ratios calculated from
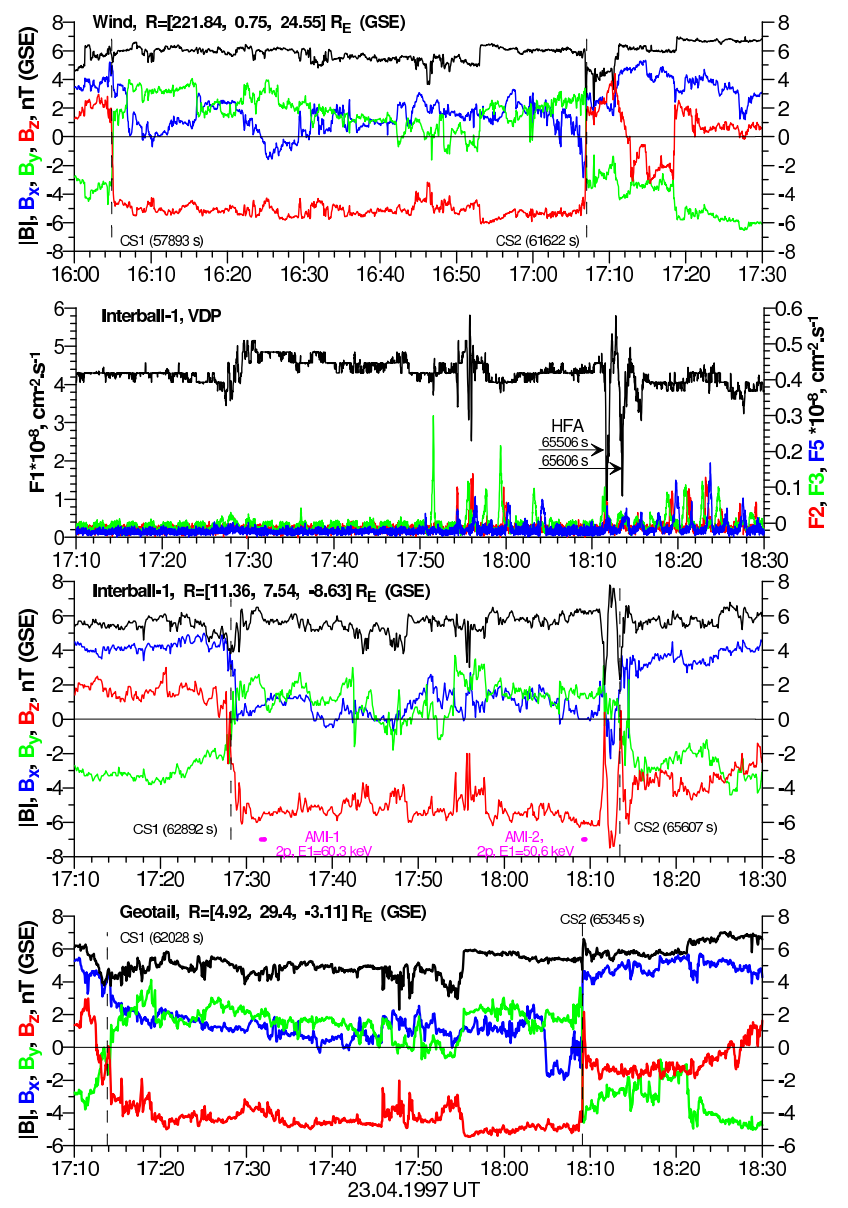

Fig. 20. Magnetic fields and solar wind fluxes (data from Wind, Interball-1 and Geotail) during two AMI events on 23 April 1997.



Fig. 21. Intersections of the CS1 and the BS during AMI-1 observation on Interball-1 on 23 April 1997 at 17:31:42-17:32:06 UT.

the densities of these ions in the solar wind plasma (enrichment in alpha-particles relative to protons). 


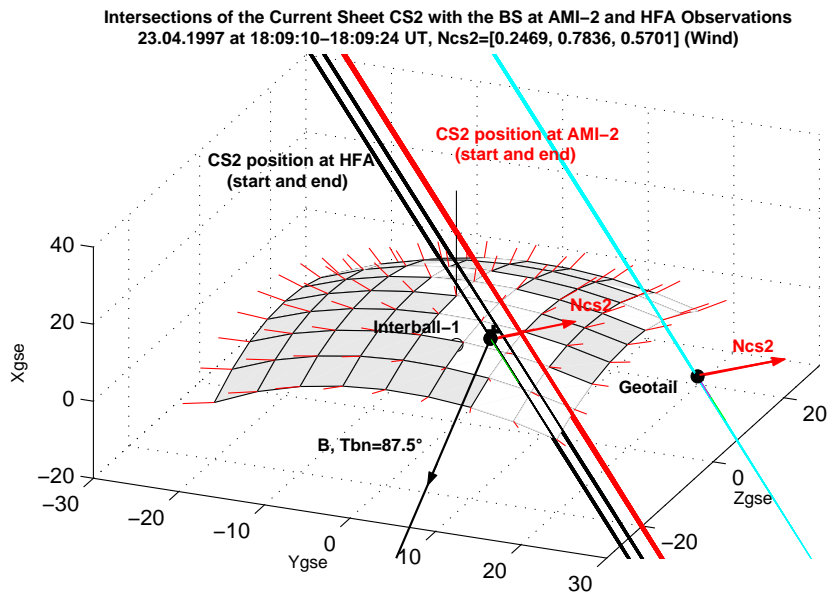

Fig. 22. Intersections of the CS2 and the BS during AMI-2 and HFA observations on Interball-1 on 23 April 1997 at 18:09:10 18:09:24 UT.
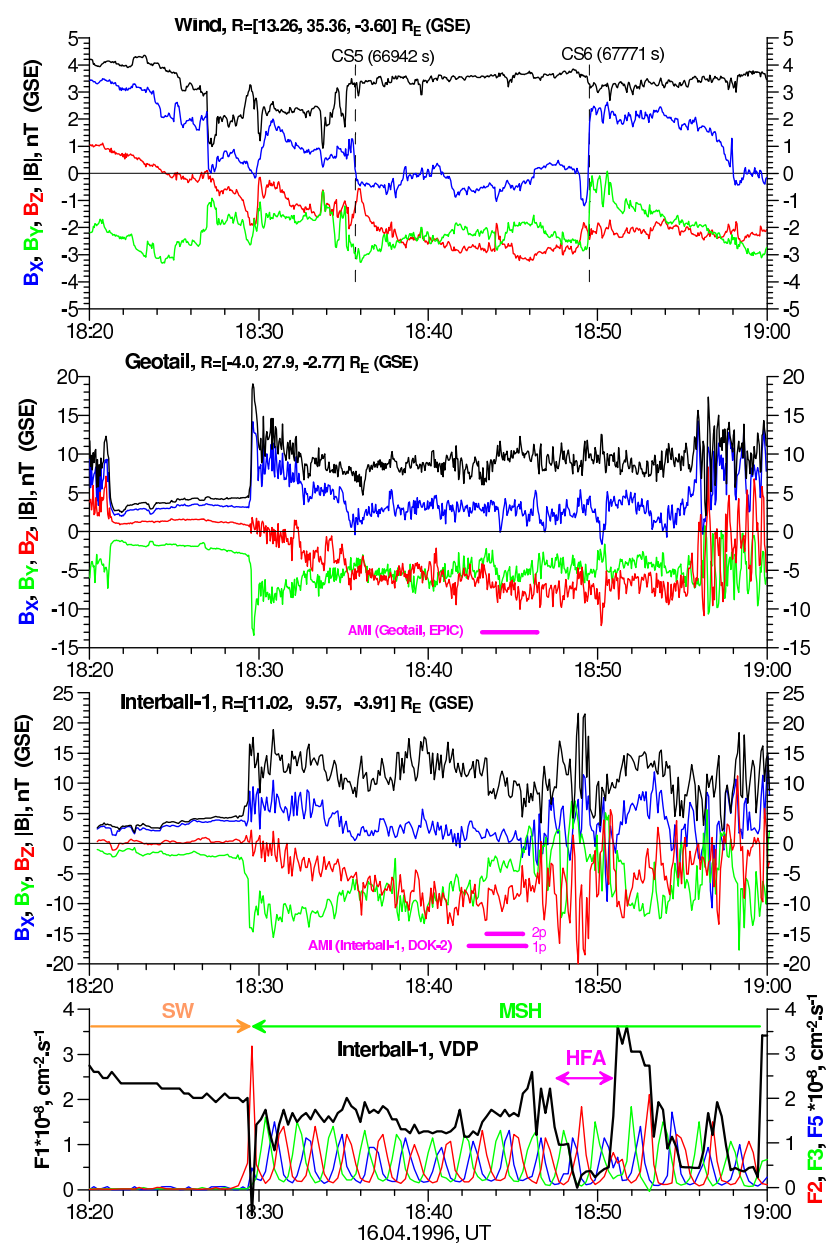

Fig. 23. Magnetic fields on Wind, Geotail, Interball-1 and plasma fluxes on Interball-1 during AMI event in the MSH on 16 April 1996.

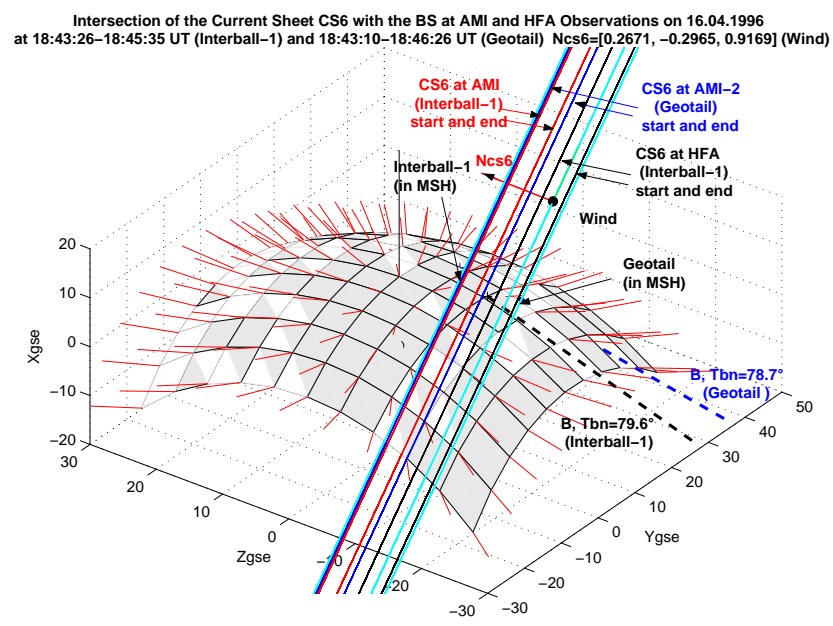

Fig. 24. Intersections of the current sheet CS6 and BS during observations of AMI and HFA on 16 April 1996 by Interball-1 (18:43:2618:45:35 UT) and Geotail (18:43:10-18:46:26 UT).

4. The study of angular distributions and temporal characteristics of AMI beams as well as the modeling of ions motion in the acceleration region showed that their acceleration occurs during a short time $(\sim 2 \mathrm{~min})$ in parts of the BS, characteristic dimensions of which don't exceed 0.8 to $1.25 R_{\mathrm{E}}$.

5. The main reason for BS current sheet disruptions was determined: It is an interplanetary current sheet (tangential discontinuity) brought by the solar wind to the BS. Under certain conditions it leads to formation of a HFA. The HFA blocks the access of the solar wind flux to some small part of the BS which stops the creation of current carriers. This leads to the current circuit disruption.

6. It was shown that the study of AMI generation process may give new information on a large-scale structure, properties, and dynamics of BS current sheet which cannot be obtained now by other known means.

7. New arguments were found in support of $\mathrm{H}$. Alfvén ideas on a great role which electric currents and electric current sheets play in space plasmas.

Acknowledgements. We are grateful to A. Lui and S. Nylund for submission of selected EPIC data for comparison with DOK-2 data, to G. N. Zastenker for provision of VDP experiment data and to A. Klassen for information on AMI observations in STEREO project before of corresponding publication. Magnetic field data from Wind and Geotail ( $3 \mathrm{~s}$ resolution) were taken from the website of Goddard Space Flight Center: http://cdaweb.gsfc.nasa.gov.

Guest Editor M. Gedalin thanks two anonymous referees for their help in evaluating this paper. 


\section{References}

Alfvén, H.: Electric Currents in Cosmic Plasmas, Revs. Geophys. Space Phys., 15, 271-284, 1977.

Anderson, K. A.: Measurements of bow shock particles far upstream from the Earth, J. Geophys. Res., 86, 4445-4454, 1981.

Klassen, A., Gómez-Herrero, R., Müller-Mellin, R., Böttcher, S., Heber, B., Wimmer-Schweingruber, R., and Mason, G. M.: STEREO/SEPT observations of upstream particle events: almost monoenergetic ion beams, Ann. Geophys., 27, 2077-2085, doi:10.5194/angeo-27-2077-2009, 2009.

Lutsenko, V. N.: Almost Monoenergetic Ions: New Support for Alfvén Ideas on the Role of Electric Currents in Space Plasmas, Phys. Chem. Earth (C), 26, 615-619, 2001.

Lutsenko, V. N. and Kudela, K.: Almost monoenergetic ions near the Earth's magnetospheric boundaries, Geophys. Res. Lett., 26, 413-415, 1999.

Lutsenko, V. N., Kudela, K., and Sarris, E. T.: The DOK-2 Experiment to Study Energetic Particles by the Tail and Auroral Probe Satellites in the Interball project, Cosmic Research, 36, 98-107, 1998.

Lutsenko, V. N., Gretchko, T. V., Kobelev, A. V., Styazhkin, V. A., and Kudela, K.: "Wavy" Energetic Ion Dispersion Events and PC-5 Type Magnetic Field Pulsations, Adv. Space Res., 30, 1783-1786, 2002.
Lutsenko, V. N., Kirpichev, I. P., Grechko, T. V., and Delcourt, D.: Source Positions for Energetic Particles Responsible for the Fine Dispersion Structures: Numerical Simulation Results, Planet. Space Sci., 53, 275-281, 2005.

Lutsenko, V. N., Gavrilova, E. A., and Grechko, T. V.: Statistics of fine dispersion structures events in energetic particle spectra: their origin and role in the outer magnetosphere, Ann. Geophys., 26, 2097-2110, doi:10.5194/angeo-26-2097-2008, 2008.

Safrankova, J., Zastenker, G., Nemecek, Z., Fedorov, A., Simersky, M., and Prech, L.: Small scale observation of magnetopause motion: preliminary results of the INTERBALL project, Ann. Geophys., 15, 562-569, doi:10.1007/s00585-997-0562-8, 1997.

Schwartz, S. J.: Hot flow anomalies near the Earth's bow shock, Adv. Space Res., 15, 107-116, 1995.

Schwartz, S. J., Paschmann, G., Sckopke, N., Bauer, T. M., Dunlop, M., Fazakerley, N., and Thomsen, M. F.: Conditions for formation of hot flow anomalies at Earth's bow shock, J. Geophys. Res., 105, 12639-12650, 2000.

Williams, D. J., McEntire, R. W., C. Schlemn II, C., Lui, A. T. Y., Gloeckler, G., Christon, S. P., and Gliem, F.: GEOTAIL energetic particles and ion composition instrument, J. Geomagn. Geoelectr., 46, 39-57, 1994. 